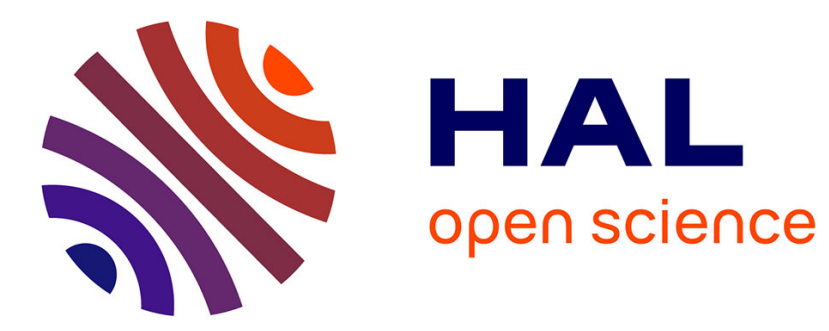

\title{
Random fields of bounded variation and computation of their variation intensity
}

Bruno Galerne

\section{To cite this version:}

Bruno Galerne. Random fields of bounded variation and computation of their variation intensity. Advances in Applied Probability, 2016, 48 (04), pp.947 - 971. 10.1017/apr.2016.60 . hal-01044582v2

\section{HAL Id: hal-01044582 \\ https://hal.science/hal-01044582v2}

Submitted on 13 Jan 2017

HAL is a multi-disciplinary open access archive for the deposit and dissemination of scientific research documents, whether they are published or not. The documents may come from teaching and research institutions in France or abroad, or from public or private research centers.
L'archive ouverte pluridisciplinaire HAL, est destinée au dépôt et à la diffusion de documents scientifiques de niveau recherche, publiés ou non, émanant des établissements d'enseignement et de recherche français ou étrangers, des laboratoires publics ou privés. 


\title{
RANDOM FIELDS OF BOUNDED VARIATION AND COMPU- TATION OF THEIR VARIATION INTENSITY
}

\author{
BRUNO GALERNE, ${ }^{*}$ Université Paris Descartes
}

\begin{abstract}
The main purpose of this paper is to define and characterize random fields of bounded variation, that is random fields with sample paths in the space of functions of bounded variation, and to study their mean total variation. Simple formulas are obtained for the mean total directional variation of random fields, based on known formulas for the directional variation of deterministic functions. It is also shown that the mean variation of random fields with stationary increments is proportional to the Lebesgue measure, and an expression of the constant of proportionality, called the variation intensity, is established. This expression shows in particular that the variation intensity only depends on the family of two-dimensional distributions of the stationary increment random field. When restricting to random sets, the obtained results give generalizations of well-known formulas from stochastic geometry and mathematical morphology. The interest of these general results is illustrated by computing the variation intensities of several classical stationary random field and random set models, namely Gaussian random fields and excursion sets, Poisson shot noises, Boolean models, dead leaves models, and random tessellations.

Keywords: Functions of bounded variation; Directional variation; Variation intensity; Specific perimeter; Stationary increment random fields; Germ-grain models;
\end{abstract}

2010 Mathematics Subject Classification: Primary 60G60

Secondary 60G17; 60G51; 60D05

\footnotetext{
* Postal address: Laboratoire MAP5 (UMR CNRS 8145), Université Paris Descartes, Sorbonne Paris Cité, 45 rue des Saints-Pères, 75006 Paris; bruno.galerne@parisdescartes.fr
} 


\section{Introduction}

This paper focuses on the definition and characterizations of random fields (r.f.) of bounded variation, that is, r.f. having their paths in the space of functions of bounded variation, as well as to provide explicit formulas for the computation of their mean total variation. Its motivations stem from the modeling of textures by r.f. in image processing where functions of bounded variation are an important model. The functional space $B V(U)$ of functions of bounded variation defined over some open set $U \subset \mathbb{R}^{d}$ is a subspace of $L^{1}(U)$ for which the functions are weakly differentiable in the sense that their distributional derivative $D f$ can be represented by a finite Radon measure over $U$ [2]. The total variation of $f \in L^{1}(U)$ is then defined by $|D f|(U)$, the total variation of the vector-valued measure $D f$, if $f \in B V(U)$, and by $+\infty$ otherwise.

Ever since the seminal paper of Rudin, Osher and Fatemi [23], the total variation has been widely used for various tasks in image processing such as denoising, zooming, deconvolution, etc. (see e.g. [3] and the references therein). Concerning textures, the total variation generally appears in the problem of decomposing on image in a cartoon part (or geometric part) and a textural part following the framework proposed by Meyer [20]. For this problem it is considered that the total variation of a textural part should be high or even infinite. Yet, to the best of our knowledge little is known on the total variation of classical texture models such as Gaussian r.f. , shot noises, dead leaves models... However, for the later germ-grain models, the r.f. correspond to random geometric images with few contours, and intuitively their total variation should depend on the geometry of the grains. Hence it is natural to ask the following question: What is the mean total variation of classical germ-grain models?

This paper provides an answer to this question by introducing general definitions and characterization results for r.f. of bounded variation. To the best of our knowledge r.f. of bounded variation over $\mathbb{R}^{d}$ have never been studied for $d \geq 2$, with the exception of the short paper [15] and the very recent paper [5] that studies the excursion sets of shot noise r.f. While the present work was motivated by image modeling, its results are presented in an arbitrary dimension $d \geq 1$ and are not limited to this field. Indeed the results are of potential interest in any subject of applied probability where piecewise smooth r.f. are at hands such as stochastic geometry (with e.g. Boolean random 
functions or colored tessellations, see Section 6.2). In comparison, the notions of regularity that are often considered in applied probability such as Hölder continuity does not allow for discontinuities in the r.f. sample paths.

Let us now detail the main results of the paper. For a r.f. $f$, our general strategy is first to deal with directional variations $\left|D_{u} f\right|, u \in S^{d-1}$, and then integrate over all directions $u \in S^{d-1}$ to obtain results on the variation $|D f|$. The advantage of dealing with directional variations is that it gives simple expressions only involving difference quotients, and it also provides additional information on the anisotropy of the r.f. $f$. The first section of this paper is devoted to deterministic functions of bounded directional variation. Relations between the directional variation $\left|D_{u} f\right|$ of a function $f$ and the integral of its difference quotients are emphasized, yielding to the fundamental relation

$$
\left|D_{u} f\right|(U)=\lim _{r \rightarrow 0} \int_{U \ominus[0, r u]} \frac{|f(x+r u)-f(x)|}{|r|} d x,
$$

for any open set $U \subset \mathbb{R}^{d}$. After this preliminary study of deterministic functions of bounded directional variation, r.f. of (locally) bounded (directional) variation are defined and characterized in Section 3. In particular, one defines the directional variation intensity measure $\Theta_{V_{u}}(f, \cdot)$ of a r.f. $f$ as the expectation of the directional variation of $f$, and it is shown that

$$
\Theta_{V_{u}}(f, U)=\mathbb{E}\left(\left|D_{u} f\right|(U)\right)=\lim _{r \rightarrow 0} \int_{U \ominus[0, r u]} \frac{\mathbb{E}(|f(x+r u)-f(x)|)}{|r|} d x .
$$

A particular interest is then given to r.f. $f$ with stationary increments having locally bounded (directional) variation in Section 4. If $f$ is such a r.f., it is proved that the mean directional variation of $f$ on every domain $U$ is proportional to the Lebesgue measure of $U$. Denoted $\theta_{V_{u}}(f)$, the constant of proportionality is called the directional variation intensity of $f$ and is given by the following practical formula:

$$
\theta_{V_{u}}(f)=\lim _{r \rightarrow 0} \frac{\mathbb{E}(|f(r u)-f(0)|)}{|r|} .
$$

In particular, the directional variation intensity $\theta_{V_{u}}(f)$ only depends on the family of two-dimensional distributions of the stationary increment r.f. $f$.

As mentioned above, we are aware of only one general result on the variation of r.f. which is due to Ibragimov [15]. Ibragimov's theorem shows that if a r.f. is Lipschitz in mean then $f$ has a.s. locally bounded variation. We improve Ibragimov's theorem 
by giving the optimal bound on the (directional) variation intensity measure and we establish that the converse of Ibragimov's theorem holds when the r.f. has stationary increments: a locally integrable stationary increment r.f. has bounded variation with a finite variation intensity if and only if it is Lipschitz in mean.

By definition, the variation of an indicator function is its perimeter [2]. Hence as a particular case of our results on the mean variation of r.f. , in Section 5 we derive formulas for the mean perimeter of random sets first established by the author in [10], and originally due to Matheron $[19,18]$. In particular, the specific directional variation of a stationary random set $X \subset \mathbb{R}^{d}$ is given by

$$
\theta_{V_{u}}(X)=2 \lim _{r \rightarrow 0} \frac{\nu_{X}(r u)}{|r|}
$$

where $\nu_{X}(y)=\mathbb{P}(y \in X, 0 \notin X)$ is the variogram of $X$. In addition, mean coarea formulas for mean total variation are established.

Finally, Section 6 illustrates the interest of the general formulas (1) and (2) by computing the variation intensity of several classical stationary r.f. models. We first study the variation of stationary Gaussian r.f and the perimeter of their excursion sets. It is shown that a Gaussian r.f. $f_{G}$ has finite variation intensity if and only if the onedimensional restrictions of its covariance are twice differentiable at 0 . According to [24], this condition also implies that the sample paths of $f_{\mathrm{G}}$ are a.s. in the Sobolev space $W_{\text {loc }}^{1,1}\left(\mathbb{R}^{d}\right)$ which shows that functions of bounded variation are not an interesting model for Gaussian r.f. The second part of Section 6 provides expressions of the directional and non directional variation intensities of several r.f. associated with germ-grain models namely: Poisson shot noise of random sets, Boolean models, colored dead leaves r.f. , and colored tessellations. A germ-grain model defines a r.f. by combining colored random sets according to an interaction principle (addition, supremum, occultation...). These constructions result in r.f. models that present numerous discontinuities along the geometric contours of the grains. For these models, the obtained formulas explicitly clarifies the somewhat intuitive relation between the geometry of the grains $X$ and the total variation of the r.f. Moreover, they show that for all the considered germ-grain models, there are only two geometric features of influence on variation intensity: the mean perimeter and the mean Lebesgue measure of the grains. 


\section{Functions of bounded directional variation and difference quotients}

This section gathers several necessary results from the theory of functions of bounded variation, with a particular interest in functions of bounded directional variation. For a general treatment of the subject we refer to the textbook of Ambrosio, Fusco and Pallara [2]. Some basic definitions are recalled and the relations between the directional variation of a function and the integral of its difference quotients are emphasized.

Notation For any open subset $U \subset \mathbb{R}^{d}, \mathcal{B}(U)$ denotes the set of Borel subsets of $U$, and we write $V \Subset U$ if $V \subset U$ is open and relatively compact in $U . L^{1}(U)$ (resp. $\left.L_{\text {loc }}^{1}(U)\right)$ denotes the space of integrable (resp. locally integrable) functions over $U$. $\mathcal{C}^{0}(U, \mathbb{R}), \mathcal{C}^{1}(U, \mathbb{R})$, and $\mathcal{C}^{\infty}(U, \mathbb{R})$ denote the space of functions from $U$ to $\mathbb{R}$ that are respectively continuous, continuously differentiable, and infinitely differentiable. The vector field spaces $\mathcal{C}^{0}\left(U, \mathbb{R}^{d}\right), \mathcal{C}^{1}\left(U, \mathbb{R}^{d}\right)$, and $\mathcal{C}^{\infty}\left(U, \mathbb{R}^{d}\right)$ are defined similarly. The spaces $\mathcal{C}_{c}^{0}(U, \mathbb{R}), \mathcal{C}_{c}^{\infty}\left(U, \mathbb{R}^{d}\right)$, etc. denote the spaces of functions or vector fields that have a compact support. $S^{d-1}$ denotes the unit Euclidean sphere in $\mathbb{R}^{d}$. If $\varphi \in \mathcal{C}^{1}(U, \mathbb{R})$ and $u \in S^{d-1}$, we write $\frac{\partial \varphi}{\partial u}(x)=\langle\nabla \varphi(x), u\rangle, x \in \mathbb{R}^{d}$, for the directional derivative of $\varphi$ in the direction $u$. $\mathcal{H}^{d-1}$ denotes the Hausdorff measure of dimension $d-1$.

Within the remaining of this section $U$ denotes an open set of $\mathbb{R}^{d}$.

Definition 1. (Functions of bounded (directional) variation.) Let $f \in L^{1}(U) . f$ is a function of bounded variation in $U$ if the distributional derivative of $f$ is representable by a finite Radon measure, i.e. if there exists a $\mathbb{R}^{d}$-valued Radon measure, noted $D f=$ $\left(D_{1} f, \ldots, D_{d} f\right)$, such that $|D f|(U)<+\infty$ and for all $\varphi=\left(\varphi_{1}, \ldots, \varphi_{d}\right) \in \mathcal{C}_{c}^{\infty}\left(U, \mathbb{R}^{d}\right)$

$$
\int_{U} f(x) \operatorname{div} \varphi(x) d x=-\sum_{i=1}^{d} \int_{U} \varphi_{i}(x) D_{i} f(d x) .
$$

Let $u \in S^{d-1}$. $f$ is a function of bounded directional variation in $U$ in the direction $u$ if the directional distributional derivative of $f$ in the direction $u$ is representable by a finite Radon measure, i.e. if there exists a signed Radon measure, noted $D_{u} f$, such that $\left|D_{u} f\right|(U)<+\infty$ and for $\varphi \in \mathcal{C}_{c}^{\infty}(U, \mathbb{R})$

$$
\int_{U} f(x) \frac{\partial \varphi}{\partial u}(x) d x=-\int_{U} \varphi(x) D_{u} f(d x)
$$


The vector space of all functions of bounded variation in $U$ (resp. bounded directional variation in $U$ in the direction $u$ ) is denoted by $B V(U)$ (resp. $B V_{u}(U)$ ).

A function $f \in L_{\mathrm{loc}}^{1}(U)$ has locally bounded variation in $U$ (resp. locally bounded directional variation in $U$ in the direction $u$ ) if $f \in B V(V)$ (resp. $f \in B V_{u}(V)$ ) for all open sets $V \Subset U$. The corresponding space is denoted by $B V_{\text {loc }}(U)$ (resp. $\left.B V_{u, \text { loc }}(U)\right)$.

If $f \in B V(U)$ then $|D f|(U)$ is called the variation of $f$ in $U$. Similarly, if $f \in$ $B V_{u}(U),\left|D_{u} f\right|(U)$ is called the directional variation of $f$ in the direction $u$ in $U$. There is of course a strong relation between the spaces $B V(U)$ and $B V_{u}(U)$. As discussed in [8] and [10, Proposition 8] for a function $f \in L^{1}(U)$ there is equivalence between (i) $f \in B V(U)$, (ii) $f \in B V_{u}(U)$ for all $u \in S^{d-1}$, and (iii) For all vectors $e_{i}$ of the canonical basis, $f \in B V_{e_{i}}(U)$. In addition, if $f \in B V(U)$ we have for all measurable sets $A \in \mathcal{B}(U)$, one has the two identities

$$
\begin{gathered}
D_{u} f(A)=\langle D f(A), u\rangle=\sum_{i=1}^{d} u_{i} D_{i} f(A), \quad u=\left(u_{1}, \ldots, u_{d}\right) \in S^{d-1}, \\
|D f|(A)=\frac{1}{2 \omega_{d-1}} \int_{S^{d-1}}\left|D_{u} f\right|(A) \mathcal{H}^{d-1}(d u)
\end{gathered}
$$

where $\omega_{d-1}$ denotes the Lebesgue measure of the unit ball in $\mathbb{R}^{d-1}$.

Remark 1. In consequence of Equation (3), the maps $u \mapsto D_{u} f(A)$ and $u \mapsto\left|D_{u} f\right|(A)$ are continuous on $S^{d-1}$ for all fixed $A \in \mathcal{B}(U)$ as soon as $f \in B V(U)$.

In what follows, given a function $f \in L^{1}(U)$ and a direction $u \in S^{d-1}$, we will consider difference quotients of the form $x \mapsto \frac{f(x+r u)-f(x)}{r}$ for some $r \neq 0$. Such functions are not defined on the whole domain $U$ but on $U \cap(-r u+U)$. Since we will typically attempt at letting $r$ tends to zero, we will restrict further this domain of definition to the one of points $x$ such that the whole segment $[x, x+r u]$ is included in $U$. This set is denoted by $U \ominus[0, r u]=\{x \in U,[x, x+r u] \subset U\}$.

Notation for difference quotient Since directional difference quotients will be central in the remaining of the paper, we adopt the following short notation

$$
f(x ; r u)=\frac{f(x+r u)-f(x)}{r}, \quad x \in U, r \in \mathbb{R} \backslash\{0\}, u \in S^{d-1},
$$

for a function or a r.f. $f$. 
Lemma 1 and Theorem 1 below extend Proposition 11 of [10] to the case of integrable functions defined over an arbitrary domain $U$ (and not only $\mathbb{R}^{d}$ as in [10]). Because of this general domain $U$, the use of standard approximation by convolution is discarded, and we thus propose adapted proofs.

Lemma 1. Let $u \in S^{d-1}$. Then for all functions $f \in B V_{u}(U)$ and $r \neq 0$,

$$
\int_{U \ominus[0, r u]}|f(x ; r u)| d x \leq\left|D_{u} f\right|(U) .
$$

Proof. The proof is an adaptation of the one of Lemma 3.24 p. 133 of [2]. Let us first suppose that $f \in \mathcal{C}^{1}(U)$. Then, one has

$$
\begin{aligned}
\int_{U \ominus[0, r]}|f(x ; r u)| d x=\int_{U \ominus[0, r u]}\left|\int_{0}^{1} \frac{\partial f}{\partial u}(x+r t u) d t\right| d x & \leq \int_{0}^{1} \int_{U \ominus[0, r u]}\left|\frac{\partial f}{\partial u}(x+r t u)\right| d x d t \\
& \leq \int_{0}^{1}\left|D_{u} f\right|(r t u+U \ominus[0, r u]) d t .
\end{aligned}
$$

Now for all $t \in[0,1], r t u+U \ominus[0, r u] \subset U$ and thus, $\left|D_{u} f\right|(r t u+U \ominus[0, r u]) \leq$ $\left|D_{u} f\right|(U)$. Hence the lemma is valid for $f \in \mathcal{C}^{1}(U)$. This inequality extends to all functions in $B V_{u}(U)$ thanks to the density of smooth functions in $B V_{u}(U)$ : for all $f \in B V_{u}(U)$ there exists a sequence $\left(f_{n}\right)_{n}, f_{n} \in \mathcal{C}^{1}(U)$, such that $\left(f_{n}\right)$ tends to $f$ in $L^{1}(U)$ and the total directional variation $\left|D_{u} f_{n}\right|(U)=\int_{U}\left|\frac{\partial f_{n}}{\partial u}\right|$ tends to $\left|D_{u} f\right|(U)$ (using the directional equivalent of [2, Theorem 3.9 p. 122]).

Let us recall the definition of weak* convergence for signed Radon measure [2, $\mathrm{p}$. 26-27]. Let $\mathcal{C}_{0}^{0}(U, \mathbb{R})$ denote the closure of the space of continuous and compactly supported functions $\mathcal{C}_{c}^{0}(U, \mathbb{R})$ for the $\sup$ norm. Then one says that a sequence $\left(\mu_{n}\right)_{n \in \mathbb{N}}$ of signed Radon measure on $U$ weak* converges to the signed Radon measure $\mu$ if for every $\varphi \in \mathcal{C}_{0}^{0}(U, \mathbb{R}), \lim _{n \rightarrow+\infty} \int_{U} \varphi(x) \mu_{n}(d x)=\int_{U} \varphi(x) \mu(d x)$. The next theorem provides a characterization of functions of finite directional variation in terms of difference quotient.

Theorem 1. Let $u \in S^{d-1}$ and $f \in L^{1}(U)$. The three following assertions are equivalent:

(i) $f \in B V_{u}(U)$.

(ii) The family of signed measures $\mu_{r}: A \mapsto \int_{U \ominus[0, r u]} f(x ; r u) \mathbb{1}_{A}(x) d x, A \in \mathcal{B}(U)$, $r \neq 0$, weak* converges to some signed Radon measure $\mu$ as $r \rightarrow 0$. 
(iii) $\liminf _{r \rightarrow 0} \int_{U \ominus[0, r u]}|f(x ; r u)| d x<+\infty$.

If any of these assertions holds, then $\mu=D_{u} f$ and

$$
\left|D_{u} f\right|(U)=\lim _{r \rightarrow 0} \int_{U \ominus[0, r u]}|f(x ; r u)| d x .
$$

Besides, Formula (5) is also valid in the degenerate case: $f$ is not in $B V_{u}(U)$ if and only if $\lim _{r \rightarrow 0} \int_{U \ominus[0, r u]}|f(x ; r u)| d x=+\infty$.

Proof. Let us begin with a preliminary observation: For all $\varphi \in \mathcal{C}_{c}^{1}(U, \mathbb{R})$,

$$
\lim _{r \rightarrow 0} \int_{U} \varphi(x) \mu_{r}(d x)=-\int_{U} \frac{\partial \varphi}{\partial u}(x) f(x) d x .
$$

Indeed, for $\varphi \in \mathcal{C}_{c}^{1}(U, \mathbb{R})$ and $r \neq 0$,

$$
\begin{aligned}
\int_{U} \varphi(x) \mu_{r}(d x) & =\int_{U \ominus[0, r u]} \varphi(x) \frac{f(x+r u)-f(x)}{r} d x \\
& =\frac{1}{r} \int_{U \ominus[-r u, 0]} \varphi(x-r u) f(x) d x-\frac{1}{r} \int_{U \ominus[0, r u]} \varphi(x) f(x) d x \\
& =\int_{U} \frac{\varphi(x-r u) \mathbb{1}_{U \ominus[-r u, 0]}(x)-\varphi(x) \mathbb{1}_{U \ominus[0, r u]}(x)}{r} f(x) d x .
\end{aligned}
$$

Hence, since for all $x \in U$,

$$
\lim _{r \rightarrow 0} \frac{\varphi(x-r u) \mathbb{1}_{U \ominus[-r u, 0]}(x)-\varphi(x) \mathbb{1}_{U \ominus[0, r u]}(x)}{r}=-\frac{\partial \varphi}{\partial u}(x)
$$

and for all $r$ such that $|r|$ is small enough such that $\operatorname{supp}(\varphi) \subset U \ominus[0, r u]$,

$\frac{\varphi(x-r u) \mathbb{1}_{U \ominus[-r u, 0]}(x)-\varphi(x) \mathbb{1}_{U \ominus[0, r u]}(x)}{r} f(x)=\frac{\varphi(x-r u)-\varphi(x)}{r} f(x) \leq\left\|\frac{\partial \varphi}{\partial u}\right\|_{\infty} f(x) \in L^{1}(U)$,

one obtains (6) by dominated convergence.

We will prove $(i) \Rightarrow(i i i) \Rightarrow(i i) \Rightarrow(i)$. $(i) \Rightarrow($ iii $)$ is immediate thanks to Lemma 1.

Let us show $(i i i) \Rightarrow(i i)$. Remark that for all $r \neq 0, \int_{U \ominus[0, r u]}|f(x ; r u)| d x=\left|\mu_{r}\right|(U)$. Since by hypothesis $\liminf _{r \rightarrow 0}\left|\mu_{r}\right|(U)<+\infty$, there exists a sequence $\left(r_{n}\right)$ converging to 0 such that $\lim _{n \rightarrow+\infty} \int_{U \ominus[0, r u]}\left|f\left(x ; r_{n} u\right)\right| d x=\liminf _{r \rightarrow 0}\left|\mu_{r}\right|(U)<+\infty$. Hence the sequence $\left(\left|\mu_{r_{n}}(U)\right|\right)_{n \in \mathbb{N}}$ is convergent, and in particular it is bounded. By weak* compactness $\left[2\right.$, Theorem 1.59 p. 26], there exists a subsequence $\left(r_{n_{k}}\right)_{k \in \mathbb{N}}$ such that $\left(\mu_{n_{n_{k}}}\right)$ weak* converges to some signed Radon measure $\mu$. Hence, by definition of the weak* convergence, for all $\psi \in \mathcal{C}_{c}^{0}(U, \mathbb{R}), \int_{U} \psi(x) \mu(d x)=\lim _{k \rightarrow+\infty} \int_{U} \psi(x) \mu_{r_{n_{k}}}(d x)$. 
But, according to (6), for all $\varphi \in \mathcal{C}_{c}^{1}(U, \mathbb{R}), \int_{U} \varphi(x) \mu(d x)=-\int_{U} \frac{\partial \varphi}{\partial u}(x) f(x) d x=$ $\lim _{r \rightarrow 0} \int_{U} \varphi(x) \mu_{r}(d x)$. By the density of $\mathcal{C}_{c}^{1}(U, \mathbb{R})$ in $\mathcal{C}_{0}^{0}(U, \mathbb{R})$ for the uniform convergence, the above equality $\int_{U} \varphi \mu=\lim \int_{U} \varphi \mu_{r}$ extends to all $\varphi \in \mathcal{C}_{0}^{0}(U, \mathbb{R})$, which shows that the whole family $\left(\mu_{r}\right)$ weak* converges to $\mu$.

Let us now show that $(i i) \Rightarrow(i)$. Since $\left(\mu_{r}\right)$ weak* converges to $\mu$ and according

to $(6)$, for all $\varphi \in \mathcal{C}_{c}^{1}(U, \mathbb{R}), \int_{U} \varphi(x) \mu(d x)=\lim _{r \rightarrow 0} \int_{U} \varphi(x) \mu_{r}(d x)=-\int_{U} \frac{\partial \varphi}{\partial u}(x) f(x) d x$ that is $f \in B V_{u}(U)$ and $D_{u} f=\mu$.

It remains to show Equation (5). Thanks to the inequality of Lemma 1, it is enough to show that $\left|D_{u} f\right|(U) \leq \liminf _{r \rightarrow 0} \int_{U \ominus[0, r u]}|f(x ; r u)| d x$. But this inequality translates in $|\mu|(U) \leq \liminf \left|\mu_{r}\right|(U)$ which is immediate since the total variation application $\nu \mapsto|\nu|(U)$ is lower-semicontinuous with respect to the weak* convergence [2, Theorem 1.59 p. 26].

\section{Random fields of bounded variation}

Within this section, $U$ denotes an open set of $\mathbb{R}^{d}$. Following the generally approved definition, a random field (r.f.) of $\mathbb{R}^{d}$ is a family of random variables $\xi_{x}:(\Omega, \mathcal{A}) \rightarrow$ $(\mathbb{R}, \mathcal{B}(\mathbb{R}))$ indexed by $x \in \mathbb{R}^{d}$. However, the study of the variation of a r.f. requires more constraint, namely that the r.f. sample paths are integrable functions.

Definition 2. (Integrable random fields.) Let $(\Omega, \mathcal{A}, \mathbb{P})$ be a probability space. An integrable random field $f$ defined on $U$ is a random element of $L^{1}(U)$, that is a measurable map $f:(\Omega, \mathcal{A}) \rightarrow\left(L^{1}(U), \mathcal{B}\left(L^{1}(U)\right)\right)$, where $L^{1}(U)$ is endowed with the Borel $\sigma$-algebra of the $L^{1}(U)$-convergence. Similarly a locally integrable random field $f$ defined on $U$ is a random variable of $L_{\text {loc }}^{1}(U)$.

We will speak of a.s. integrable random field to allow the function $f$ to be outside $L^{1}(U)$ with probability 0 . Let us observe that if $f$ is an integrable r.f., then the integrals $\omega \mapsto \int_{\mathbb{R}^{d}} f(\omega, x) \varphi(x) d x, \varphi \in \mathcal{C}_{c}^{0}\left(\mathbb{R}^{d}, \mathbb{R}\right)$, are well-defined random variables and thus $f \mathcal{L}^{d}$ defines a unique signed Radon measure. Let us also remark that if $f:\left(\Omega \times \mathbb{R}^{d}, \mathcal{A} \otimes \mathcal{B}\left(\mathbb{R}^{d}\right)\right) \rightarrow(\mathbb{R}, \mathcal{B}(\mathbb{R}))$ is a jointly measurable r.f. such that its sample paths are almost surely integrable, then $f$ defines an integrable r.f. in the sense of the above definition 
Definition 3. (Random fields of bounded (directional) variation.) An a.s. integrable r.f. $f \in L^{1}(U)$ is a random field of bounded variation in $U$ if there exists some random $\mathbb{R}^{d}$-valued Radon measure $D f=\left(D_{1} f, \ldots, D_{d} f\right)$ such that $|D f|(U)$ is a.s. finite and for all $\varphi=\left(\varphi_{1}, \ldots, \varphi_{d}\right) \in \mathcal{C}_{c}^{\infty}\left(U, \mathbb{R}^{d}\right)$,

$$
\int_{U} f(x) \operatorname{div} \varphi(x) d x=-\sum_{i=1}^{d} \int_{U} \varphi_{i}(x) D_{i} f(d x) \text { a.s. }
$$

Let $u \in S^{d-1}$. An a.s. integrable r.f. $f \in L^{1}(U)$ is a r.f. of bounded directional variation in $U$ in the direction $u$ if there exists some random signed Radon measure $D_{u} f$ such that $\left|D_{u} f\right|(U)$ is a.s. finite and for all $\varphi \in \mathcal{C}_{c}^{\infty}(U, \mathbb{R})$,

$$
\int_{\mathbb{R}^{d}} f(x) \frac{\partial \varphi}{\partial u}(x) d x=-\int_{\mathbb{R}^{d}} \varphi(x) D_{u} f(d x) .
$$

An a.s. locally integrable r.f. $f \in L_{\mathrm{loc}}^{1}(U)$ is a r.f. of locally bounded variation in $U$ (resp. r.f. of bounded directional variation in $U$ in the direction $u$ ) if for all $V \Subset U$ the restriction of $f$ to $V$ is a r.f. of bounded variation in $V$ (resp. of bounded directional variation in $V$ in the direction $u$ ).

Definition 4. ((Directional) variation intensity measures.) For a r.f. $f$ of locally bounded variation in $U$, the intensity measure of the variation $|D f|$, that is the measure $A \mapsto \mathbb{E}(|D f|(A))$, is called the variation intensity measure of $f$ and is denoted $\Theta_{V}(f, \cdot)$. Similarly, for a r.f. $f$ of locally bounded directional variation in $U$ in the direction $u \in S^{d-1}$, the intensity measure of the variation $\left|D_{u} f\right|$, that is the measure $A \mapsto \mathbb{E}\left(\left|D_{u} f\right|(A)\right)$, is called the directional variation intensity measure of $f$ in the direction $u$ and is denoted $\Theta_{V_{u}}(f, \cdot)$.

The variation intensity measure is an important characteristic of a r.f. $f$ of bounded variation: for all measurable sets $A, \Theta_{V}(f, A)$ is the mean variation of $f$ in $A$. The mean variation $\Theta_{V}(f, U)$ on the whole domain $U$ will be called the mean total variation of $f$ and $\Theta_{V_{u}}(f, U)$ will be called the mean total directional variation of $f$ in the direction $u$. One easily establishes the analog of Equation (4) for the case of r.f. of bounded variation.

Proposition 1. (Integral geometric formula for directional variation intensity measures.) Let $f$ be a r.f. of bounded variation in $U$. Then $f$ is a r.f. of bounded 
directional variation in $U$ for all directions $u \in S^{d-1}$, and for all $A \in \mathcal{B}(U)$,

$$
\Theta_{V}(f, A)=\frac{1}{2 \omega_{d-1}} \int_{S^{d-1}} \Theta_{V_{u}}(f, A) \mathcal{H}^{d-1}(d u) .
$$

Proof. As for Proposition 8 of [10], if $D f$ is a random $\mathbb{R}^{d}$-valued Radon measure representing the distributional derivative of $f$ then $\langle D f, u\rangle$ is a random signed Radon measure which represents the directional distributional derivative of $f$. Hence $f$ is a r.f. of bounded directional variation in $U$ for all directions $u \in S^{d-1}$. The integral geometric formula is obtained in applying Fubini theorem to Formula (4).

The next proposition shows that the proposed definition of r.f. of bounded variation is equivalent with a more instinctive one: a r.f. has bounded variation if its sample paths have a.s. bounded variation.

Proposition 2. (Sample paths of r.f. of bounded variation.) Let $f: \Omega \times U \rightarrow \mathbb{R}$ be $a$ jointly measurable r.f. such that $f(\omega, \cdot) \in L^{1}(U)$ a.s. and let $u \in S^{d-1}$. Then $f$ is a r.f. of bounded directional variation in $U$ in the direction $u$ (in the sense of Definition 3) if and only if its sample paths $x \mapsto f(\omega, x)$ are in $B V_{u}(U)$ for $\mathbb{P}$-a.e. $\omega \in \Omega$. Similarly, $f$ is a r.f. of bounded variation in $U$ if and only if its sample paths $x \mapsto f(\omega, x)$ are in $B V(U)$ for $\mathbb{P}$-a.e. $\omega \in \Omega$.

Proof. Let us first show the equivalence for r.f. of bounded directional variation. The direct sense is immediate from the definition: since $D_{u} f$ is a.s. a Radon measure, the sample paths are a.s. in $B V_{u}(U)$. Conversely, note $\Omega^{\prime} \subset \Omega$ the set of $\omega \in \Omega$ for which $x \mapsto f(\omega, x)$ are in $B V_{u}(U)$. Then, for all $\omega \in \Omega^{\prime}$, there exists a signed Radon measure $\mu(\omega, \cdot)$ such that for all $\varphi \in \mathcal{C}_{c}^{\infty}(U, \mathbb{R}), \int_{U} f(\omega, x) \frac{\partial \varphi}{\partial u}(x) d x=-\int_{U} \varphi(x) \mu(\omega, d x)$. The only difficulty is to ensure that $\omega \mapsto \mu(\omega, \cdot)$ is a well-defined random signed Radon measure, that is a measurable map. Let $\left(r_{n}\right)$ be a sequence converging to 0 . According to Theorem 1, for all $\omega \in \Omega^{\prime}, \mu(\omega, \cdot)$ is the weak* limit of the sequence of signed Radon measures

$$
\mu_{r_{n}}(\omega, A)=\int_{U \ominus[0, r u]} \frac{f\left(\omega, x+r_{n} u\right)-f(\omega, x)}{r_{n}} \mathbb{1}_{A}(x) d x, \quad A \in \mathcal{B}(U) .
$$

Hence $\mu$ is measurable since it is the a.s. limit of the weak* convergent sequence of random signed Radon measures $\left(\mu_{n}\right)_{n \in \mathbb{N}}$. The corresponding equivalence for r.f. of 
bounded variation is straightforward using the fact that $f(\omega, \cdot) \in B V(U)$ if and only if $B V_{e_{i}}(U)$ for all $e_{i}$ in the canonical basis.

Remark 2. (Notation.) Proposition 2 extends directly to r.f. of locally bounded variation and locally bounded directional variation. In view of Proposition 2 we will use the notation $f \in B V(U)$ a.s., $f \in B V_{u, \text { loc }}(U)$ a.s., etc. to express that a r.f. $f$ has bounded variation, locally bounded directional variation, etc.

Let us transcript Theorem 1 in the case of r.f. to establish an integral expression of the total variation intensity.

Proposition 3. (Characterization of r.f. of bounded directional variation.) Let $f \in$ $L^{1}(U)$ a.s. and $u \in S^{d-1}$. Then the three following assertions are equivalent:

(i) $f \in B V_{u}(U)$ a.s.

(ii) $\liminf _{r \rightarrow 0} \int_{U \ominus[0, r u]}|f(x ; r u)| d x<+\infty$ a.s.

(iii) $\lim _{r \rightarrow 0} \int_{U \ominus[0, r u]}|f(x ; r u)| d x$ exists and is finite a.s.

If any of these conditions holds, then the total variation intensity is

$$
\Theta_{V_{u}}(f, U)=\lim _{r \rightarrow 0} \int_{U \ominus[0, r u]} \mathbb{E}(|f(x ; r u)|) d x .
$$

Proof. The characterization is a consequence of Theorem 1. Let us now turn to the expression of the total variation intensity. According to Theorem $1, \lim _{r \rightarrow 0} \int_{U \ominus[0, r u]}|f(x ; r u)| d x=$ $\left|D_{u} f\right|(U)$ a.s. and by Lemma 1 for all $r \neq 0, \int_{U \ominus[0, r u]}|f(x ; r u)| d x \leq\left|D_{u} f\right|(U)$ a.s. Hence if the r.v. $\left|D_{u} f\right|(U)$ is integrable, Formula (7) is obtained by dominated convergence and Fubini theorem. If $\mathbb{E}\left(\left|D_{u} f\right|(U)\right)=\Theta_{V_{u}}(f, U)=+\infty$, then Formula (7) is still valid since by Fatou lemma

$$
+\infty=\Theta_{V_{u}}(f, U)=\mathbb{E}\left(\liminf _{r \rightarrow 0} \int_{U \ominus[0, r u]}|f(x ; r u)| d x\right) \leq \liminf _{r \rightarrow 0} \mathbb{E}\left(\int_{U \ominus[0, r u]}|f(x ; r u)| d x\right) .
$$

Remark 3. (Degenerate case.) Formula (7) can be extended to the degenerate case where the limit on the right-hand side is infinite. However let us specify that this degenerate case is more subtle than in the deterministic case (see Theorem 1). Indeed there are two different cases for which the limit of Formula (7) is infinite: either the 
r.f. $f$ is not of bounded variation or $f$ is of bounded variation but its total variation $\left|D_{u} f\right|(U)$ has infinite expectation. In both cases, it is coherent to say that the mean total variation of the process is infinite. This convention will be used in the remaining of the paper.

As mentioned in the introduction, we are aware of only one result dealing with the variation of r.f. defined over $\mathbb{R}^{d}$ for $d \geq 2$. This result due to Ibragimov [15] gives a sufficient condition for a r.f. to be of bounded variation.

Theorem 2. (Ibragimov's theorem [15].) Let $U \Subset \mathbb{R}^{d}$ and let $f: \Omega \times U \rightarrow \mathbb{R}$ be a measurable and separable random field. Suppose that there exists $x_{0} \in U$ such that $\mathbb{E}\left(f\left(x_{0}\right)\right)<+\infty$ and that there exists $K>0$ such that for any $x, y \in U$, $\mathbb{E}(|f(x)-f(y)|) \leq K|x-y|$. Then the realizations of $f$ are a.s. in $B V(U)$, and there is a constant $C>0$ such that for all $A \in \mathcal{B}(U) \Theta_{V}(f, A) \leq C \mathcal{L}^{d}(A)$.

Let us establish results similar to Ibragimov's theorem for both r.f. of bounded variation and r.f. of bounded directional variation.

Proposition 4. (A sufficient condition for locally bounded directional variation.) Let $f: \Omega \times U \rightarrow \mathbb{R}$ be a jointly measurable r.f. such that $f(\omega, \cdot) \in L_{\text {loc }}^{1}(U)$ a.s.

(i) (directional case) Let $u \in S^{d-1}$. Suppose that there exists a constant $K>0$ such that for all $r \in \mathbb{R}$ and $x \in U \ominus[0, r u]$,

$$
\mathbb{E}(|f(x+r u)-f(x)|) \leq K|r| .
$$

Then $f$ is a r.f. of locally bounded directional variation in the direction $u$ and for all $W \Subset U, \Theta_{V_{u}}(f, W) \leq K \mathcal{L}^{d}(W)$.

(ii) (non directional case) Suppose that there exists a constant $K>0$ such that for all $x, y \in U, \mathbb{E}(|f(x)-f(y)|) \leq K|x-y|$. Then $f$ is a r.f. of locally bounded variation, and for all $W \Subset U, \Theta_{V}(f, W) \leq \frac{d \omega_{d}}{2 \omega_{d-1}} K \mathcal{L}^{d}(W)$.

Proof. Let $W \Subset U$. By Fatou lemma and Fubini theorem

$$
\begin{aligned}
\mathbb{E}\left(\liminf _{r \rightarrow 0} \int_{W \ominus[0, r u]}|f(x ; r u)| d x\right) & \leq \liminf _{r \rightarrow 0} \mathbb{E}\left(\int_{W \ominus[0, r u]}|f(x ; r u)| d x\right) \\
& \leq K \liminf _{r \rightarrow 0} \mathcal{L}^{d}(W \ominus[0, r u]) \leq K \mathcal{L}^{d}(W)<+\infty
\end{aligned}
$$


In particular, $\liminf \int_{W \ominus[0, r u]}|f(x ; r u)| d x<+\infty$ a.s. and thus $f \in B V_{u}(W)$ a.s. by Proposition 3. This is valid for all $W \Subset U$, and thus $f \in B V_{u, \text { loc }}(U)$ a.s. The inequality $\Theta_{V_{u}}(f, W) \leq K \mathcal{L}^{d}(W)$ is immediate using Formula $(7)$.

Now for the non directional case, if for all $x, y \in U, \mathbb{E}(|f(x)-f(y)|) \leq K|x-y|$, then the first part of the proposition shows that $f$ has a.s. bounded directional variation in the $d$ directions of the canonical basis, and thus $f \in B V(U)$ a.s. In addition, for all $W \Subset U$ and for all $u \in S^{d-1}, \Theta_{V_{u}}(f, W) \leq K \mathcal{L}^{d}(W)$. Hence, by Proposition 1, for all $U \Subset \mathbb{R}^{d}$,

$$
\Theta_{V}(f, W)=\frac{1}{2 \omega_{d-1}} \int_{S^{d-1}} \Theta_{V_{u}}(f, W) \mathcal{H}^{d-1}(d u) \leq \frac{d \omega_{d}}{2 \omega_{d-1}} K \mathcal{L}^{d}(W),
$$

where we used that $\mathcal{H}^{d-1}\left(S^{d-1}\right)=d \omega_{d}$.

In the following, Property (8) will be referred to as Lipschitzness in mean in the direction $u$. Let us specify that the upper bounds given in Proposition 4 are optimal. Indeed, as it will be shown later (see Corollary 1), any r.f. $f$ with stationary increments and having locally bounded directional variation satisfies $\Theta_{V_{u}}(f, W)=K \mathcal{L}^{d}(W)$ for some constant $K$ (and the upper bound on $\Theta_{V}(f, W)$ is shown to be reached for isotropic r.f. with stationary increments).

\section{Variation intensity of random fields with stationary increments}

Let $f: \Omega \times \mathbb{R}^{d} \rightarrow \mathbb{R}$ be a jointly measurable r.f. $f$ is said to have stationary increments or to be a stationary increment random field, if for all $y \in \mathbb{R}^{d}$, both r.f. $x \mapsto f(x+y)-f(y)$ and $x \mapsto f(x)-f(0)$ have the same finite-dimensional distributions. The next theorem, which constitutes the main result of the paper, defines and gives an expression of the directional variation intensity $\theta_{V_{u}}(f)$ of a stationary increment r.f. $f$.

Theorem 3. (Definition and computation of the (directional) variation intensity of stationary increment r.f..) Let $u \in S^{d-1}$ and let $f: \Omega \times \mathbb{R}^{d} \rightarrow \mathbb{R}$ be a jointly measurable stationary increment r.f. such that $f \in L_{\text {loc }}^{1}\left(\mathbb{R}^{d}\right)$ a.s. The following assertions are equivalent:

(i) $f \in B V_{u, \text { loc }}\left(\mathbb{R}^{d}\right)$ a.s. and its directional variation intensity measure $\Theta_{V_{u}}(f, \cdot)$ is locally finite. 
(ii) $f \in B V_{u, \text { loc }}\left(\mathbb{R}^{d}\right)$ a.s. and its directional variation intensity measure $\Theta_{V_{u}}(f, \cdot)$ is proportional to the Lebesgue measure: there exists a constant $\theta_{V_{u}}(f) \geq 0$ such that for all $A \in \mathcal{B}\left(\mathbb{R}^{d}\right), \Theta_{V_{u}}(f, A)=\theta_{V_{u}}(f) \mathcal{L}^{d}(A)$.

(iii) $\liminf _{r \rightarrow 0} \mathbb{E}(|f(0 ; r u)|)<+\infty$.

(iv) $\lim _{r \rightarrow 0} \mathbb{E}(|f(0 ; r u)|)$ exists and is finite.

If any of the above assertions holds, the constant of proportionality $\theta_{V_{u}}(f)$ is given by

$$
\theta_{V_{u}}(f)=\liminf _{r \rightarrow 0} \mathbb{E}(|f(0 ; r u)|)=\lim _{r \rightarrow 0} \mathbb{E}(|f(0 ; r u)|)
$$

and is called the directional variation intensity of $f$ in the direction $u$.

Regarding non directional variation, $f \in B V_{\mathrm{loc}}\left(\mathbb{R}^{d}\right)$ a.s. with a locally finite variation intensity measure $\Theta_{V}(f, \cdot)$ if and only if for all $u \in S^{d-1}$ the limit $\lim _{r \rightarrow 0} \mathbb{E}(|f(0 ; r u)|)$ exists and is finite. In this case the variation intensity measure $\Theta_{V}(f, \cdot)$ is proportional to the Lebesgue measure, and the constant of proportionality $\theta_{V}(f)$, called the variation intensity of $f$, is given by

$$
\begin{aligned}
\theta_{V}(f)=\frac{1}{2 \omega_{d-1}} \int_{S^{d-1}} \theta_{V_{u}}(f) \mathcal{H}^{d-1}(d u) & =\frac{1}{2 \omega_{d-1}} \int_{S^{d-1}} \lim _{r \rightarrow 0} \mathbb{E}(|f(0 ; r u)|) \mathcal{H}^{d-1}(d u) \\
& =\lim _{r \rightarrow 0} \frac{1}{2 \omega_{d-1}} \int_{S^{d-1}} \mathbb{E}(|f(0 ; r u)|) \mathcal{H}^{d-1}(d u) .
\end{aligned}
$$

Proof. We will show the following chain of implications: (ii) $\Leftrightarrow$ (i) $\Rightarrow$ (iv) $\Rightarrow$ (iii) $\Rightarrow$ (i). First remark that (iv) $\Rightarrow$ (iii) and (ii) $\Rightarrow$ (i) are trivial. Remark that since $f$ has stationary increments, thanks to Fubini theorem for all $U \Subset \mathbb{R}^{d}$ and $r \neq 0$,

$$
\mathbb{E}\left(\int_{U \ominus[0, r u]}|f(x ; r u)| d x\right)=\mathbb{E}(|f(0 ; r u)|) \mathcal{L}^{d}(U \ominus[0, r u]) .
$$

Let us show (i) $\Rightarrow$ (iv) and (i) $\Rightarrow$ (ii). Suppose that $f \in B V_{u, \text { loc }}\left(\mathbb{R}^{d}\right)$ a.s. and that $\Theta_{V_{u}}(f, \cdot)$ is locally finite. Let $U \Subset \mathbb{R}^{d}$. According to Lemma $1, \int_{U \ominus[0, r u]}|f(x ; r u)| d x \leq$ $\left|D_{u} f\right|(U)$. By hypothesis, $\left|D_{u} f\right|(U)$ is an $L^{1}$-r.v. Hence, one can apply the reverse 
Fatou lemma,

$$
\begin{aligned}
\limsup _{r \rightarrow 0} \mathbb{E}(|f(0 ; r u)|) \mathcal{L}^{d}(U) & =\limsup _{r \rightarrow 0} \mathbb{E}(|f(0 ; r u)|) \mathcal{L}^{d}(U \ominus[0, r u]) \\
& =\limsup _{r \rightarrow 0} \mathbb{E}\left(\int_{U \ominus[0, r u]}|f(x ; r u)| d x\right) \\
& \leq \mathbb{E}\left(\limsup _{r \rightarrow 0} \int_{U \ominus[0, r u]}|f(x ; r u)| d x\right) \\
& \leq \mathbb{E}\left(\left|D_{u} f\right|(U)\right)=\Theta_{V_{u}}(f, U)<+\infty .
\end{aligned}
$$

Besides, by Fatou lemma,

$$
\begin{aligned}
\Theta_{V_{u}}(f, U)=\mathbb{E}\left(\left|D_{u} f\right|(U)\right) & =\mathbb{E}\left(\liminf _{r \rightarrow 0} \int_{U \ominus[0, r u]}|f(x ; r u)| d x\right) \\
& \leq \liminf _{r \rightarrow 0} \mathbb{E}(|f(0 ; r u)|) \mathcal{L}^{d}(U \ominus[0, r u]) \\
& \leq \liminf _{r \rightarrow 0} \mathbb{E}(|f(0 ; r u)|) \mathcal{L}^{d}(U)
\end{aligned}
$$

Hence we have shown that for all $U \Subset \mathbb{R}^{d}$,

$$
\limsup _{r \rightarrow 0} \mathbb{E}(|f(0 ; r u)|) \mathcal{L}^{d}(U) \leq \Theta_{V_{u}}(f, U) \leq \liminf _{r \rightarrow 0} \mathbb{E}(|f(0 ; r u)|) \mathcal{L}^{d}(U) .
$$

This shows that $\mathbb{E}(|f(0 ; r u)|)$ has a finite limit, say $\theta_{V_{u}}(f)$, and that for all bounded open sets $U, \Theta_{V_{u}}(f, U)=\theta_{V_{u}}(f) \mathcal{L}^{d}(U)$. This equality extends to all Borel sets $A \in$ $\mathcal{B}\left(\mathbb{R}^{d}\right)$ and thus $\Theta_{V_{u}}$ is proportional to the Lebesgue measure.

It remains to show (iii) $\Rightarrow$ (i). Thanks to Fatou lemma and Equation (10),

$$
\begin{aligned}
\mathbb{E}\left(\liminf _{r \rightarrow 0} \int_{U \ominus[0, r u]}|f(x ; r u)| d x\right) & \leq \liminf _{r \rightarrow 0} \mathbb{E}(|f(0 ; r u)|) \mathcal{L}^{d}(U \ominus[0, r u]) \\
& \leq \mathcal{L}^{d}(U) \liminf _{r \rightarrow 0} \mathbb{E}(|f(0 ; r u)|)<+\infty
\end{aligned}
$$

In particular, $\lim \inf _{r \rightarrow 0} \int_{U \ominus[0, r u]}|f(x ; r u)| d x$ is almost surely finite, and thus by Proposition $3 f \in B V_{u}(U)$ a.s. This is valid for all $U \Subset \mathbb{R}^{d}$, and thus $f \in B V_{u, \text { loc }}\left(\mathbb{R}^{d}\right)$ a.s. Besides, by Formula (5), the above equation reads $\Theta_{V_{u}}(f, U) \leq \liminf _{r \rightarrow 0} \mathbb{E}(|f(0 ; r u)|) \mathcal{L}^{d}(U)<$ $+\infty$, and thus $\Theta_{V_{u}}(f, \cdot)$ is locally finite.

Regarding non directional variation, the results is a straightforward consequence of the above equivalence and Proposition 1 which becomes Formula (9) in this context. The fact that the limit and the integral commute follows from the bounded convergence 
theorem using the bound

$$
\mathbb{E}(|f(0 ; r u)|) \leq \theta_{V_{u}}(f) \frac{\mathcal{L}^{d}(B(0,1))}{\mathcal{L}^{d}(B(0,1) \ominus[0, r u])} \leq \theta_{V_{u}}(f) \frac{1}{(1-R)^{d}}, \quad r \in[-R, R] \backslash\{0\}, \quad R<1,
$$

given by Lemma 1 and Theorem 3 .

Remark 4. (Degenerate Case.) As for Proposition 3, one extends the definition of $\theta_{V_{u}}(f)$ to the degenerate case where $\lim _{r \rightarrow 0} \mathbb{E}(|f(0 ; r u)|)=+\infty$.

Let us remark that the directional variation intensities $\theta_{V_{u}}(f), u \in S^{d-1}$, and the variation intensity $\theta_{V}(f)$ only depend on the two-dimensional distributions of the stationary increment r.f. $f$. In addition, if the r.f. $f$ has almost surely $\mathcal{C}^{1}$ sample paths, then one simply has $\theta_{V}(f)=\mathbb{E}(\|\nabla f(0)\|)$ and $\theta_{V_{u}}(f)=\mathbb{E}(|\langle\nabla f(0), u\rangle|)$.

To finish this section, it is shown that the directional Lipschitzness in mean introduced in Section 3 is a necessary and sufficient condition for stationary increment r.f. having finite directional variation intensity.

Corollary 1. (Directional Lipschitzness in mean of stationary increment r.f..) Let $u \in S^{d-1}$ and $f: \Omega \times \mathbb{R}^{d} \rightarrow \mathbb{R}$ be a jointly measurable stationary increment $r$.f. such that $f \in L_{\mathrm{loc}}^{1}\left(\mathbb{R}^{d}\right)$ a.s. Then for all $x \in \mathbb{R}^{d}$ and $r \in \mathbb{R}, \mathbb{E}(|f(x+r u)-f(x)|) \leq \theta_{V_{u}}(f)|r|$.

Proof. First, since $f$ has stationary increments, for all $x \in \mathbb{R}^{d}$ and $r \in \mathbb{R}, \mathbb{E}(|f(x+r u)-f(x)|)=$ $\mathbb{E}(|f(r u)-f(0)|)$. Let $r \neq 0$ and $\rho>0$. By Lemma 1 ,

$$
\int_{B(0, \rho) \ominus[0, r u]}|f(x ; r u)| d x \leq\left|D_{u} f\right|(B(0, \rho)) \text { a.s. }
$$

Hence, by Fubini theorem $\mathbb{E}(|f(0 ; r u)|) \mathcal{L}^{d}(B(0, \rho) \ominus[0, r u]) \leq \theta_{V_{u}}(f) \mathcal{L}^{d}(B(0, \rho))$ and thus, $\mathbb{E}(|f(0 ; r u)|) \leq \theta_{V_{u}}(f) \frac{\mathcal{L}^{d}(B(0, \rho))}{\mathcal{L}^{d}(B(0, \rho) \ominus[0, r u])}$. Letting $\rho \rightarrow+\infty$ one obtains the announced inequality.

Remark 5. Combining the results of Proposition 4 and Corollary 1 we obtain that a stationary increment r.f. has finite directional variation intensity if and only if it is directionally Lipschitz in mean. Let us also mention that a local Lipschitzness in mean in a neighborhood of the origin is sufficient. Indeed, if there exists a constant $K>0$ and a real $R>0$ such that for all $r \in[0, R), \mathbb{E}(|f(r u)-f(0)|) \leq K|r|$ then $\theta_{V_{u}}(f)=\liminf _{r \rightarrow 0} \mathbb{E}(|f(0 ; r u)|) \leq K<+\infty$. 
As already mentioned in Section 3, Corollary 1 shows that the first upper bound of Proposition 4 becomes an equality for any stationary increment r.f. of bounded directional variation having a finite directional variation intensity.

\section{The case of random sets}

In this section we apply the general results of the previous sections to the special case of random indicator functions. This enables to obtain all the results of [10] linking the covariogram and the mean perimeter of sets as direct corollaries (let us recall that these formulas were first established by Matheron and are widely stated in the mathematical morphology literature $[19,26,18])$.

The classical framework for the study of random sets is the one of random closed sets (RACS) [21]. The framework of random integrable functions gives another framework for random sets, namely the one of random measurable sets (RAMS). We refer to [12] for a discussion regarding the links between RAMS and RACS. By definition a RAMS $X$ is a measurable map $X:(\Omega, \mathcal{A}) \rightarrow\left(\mathcal{B}\left(\mathbb{R}^{d}\right), \mathcal{B}\left(\mathcal{B}\left(\mathbb{R}^{d}\right)\right)\right)$ where $\mathcal{B}\left(\mathcal{B}\left(\mathbb{R}^{d}\right)\right)$ denotes the Borel $\sigma$-algebra induced by the local convergence in measure (The local convergence in measure of a sequence $(A)_{n}$ towards $A$ simply corresponds to the convergence in $L_{\text {loc }}^{1}\left(\mathbb{R}^{d}\right)$ of the indicator functions $\mathbb{1}_{A_{n}}$ towards $\left.\mathbb{1}_{A}\right)$. Let us recall that the (variational) perimeter $\operatorname{Per}(A)$ of a measurable set $A$ is defined as $\operatorname{Per}(A)=\left|D \mathbb{1}_{A}\right|\left(\mathbb{R}^{d}\right)$ if $\mathbb{1}_{A} \in$ $B V\left(\mathbb{R}^{d}\right)$, and $\operatorname{Per}(A)+\infty$ otherwise. Similarly the directional variation $V_{u}(A)$ in the direction $u \in S^{d-1}$ of $A$ is $V_{u}(A)=\left|D_{u} \mathbb{1}_{A}\right|\left(\mathbb{R}^{d}\right)$ if $\mathbb{1}_{A} \in B V_{u}\left(\mathbb{R}^{d}\right)$ and $+\infty$ otherwise.

Let us first consider a RAMS $X$ for which $\mathbb{E}\left(\mathcal{L}^{d}(X)\right)<+\infty$. The mean covariogram $\gamma_{X}$ of $X$ is the function $\gamma_{X}: \mathbb{R}^{d} \rightarrow[0, \infty[$ defined by

$$
\gamma_{X}(y)=\mathbb{E}\left(\mathcal{L}^{d}(X \cap(y+X))\right)=\int_{\Omega \times \mathbb{R}^{d}} \mathbb{1}_{X(\omega)}(x) \mathbb{1}_{X(\omega)}(x+y) \mathbb{P}(d \omega) d x .
$$

Thanks to the identity $\gamma_{X}(0)-\gamma_{X}(y)=\frac{1}{2} \mathbb{E}\left(\int_{\mathbb{R}^{d}}\left|\mathbb{1}_{X}(x+y)-\mathbb{1}_{X}(x)\right| d x\right)$ due to Matheron [10], the identity of Proposition 3 becomes

$$
\lim _{r \rightarrow 0} \frac{\gamma_{X}(0)-\gamma_{X}(r u)}{|r|}=\frac{1}{2} \mathbb{E}\left(V_{u}(X)\right) .
$$

Noting $\left(\gamma_{X}^{u}\right)^{\prime}(0)=\lim _{r \rightarrow 0^{+}} \frac{\gamma_{X}(r u)-\gamma_{X}(0)}{r}$, by integration over all the directions $u \in$ 
$S^{d-1}$ and by applying the dominated convergence theorem one has

$$
-\frac{1}{\omega_{d-1}} \int_{S^{d-1}}\left(\gamma_{X}^{u}\right)^{\prime}(0) \mathcal{H}^{d-1}(d u)=\mathbb{E}(\operatorname{Per}(X)) .
$$

Hence the main results of [10] are also valid in the more general framework of RAMS (see also [12] for equivalent formulas for local covariogram).

Let us now discuss the case of stationary random sets. We call stationary any random set $X: \Omega \rightarrow \mathcal{B}\left(\mathbb{R}^{d}\right)$ such that the map $(\omega, x) \mapsto \mathbb{1}_{X(\omega)}(x)$ is a jointly measurable and stationary r.f. A stationary random set is said to be of locally bounded variation if the stationary r.f. $\mathbb{1}_{X}:(\omega, x) \mapsto \mathbb{1}_{X(\omega)}(x)$ is of locally bounded variation, and one defines similarly random sets of locally bounded directional variation. One writes $\theta_{V}(X):=\theta_{V}\left(\mathbb{1}_{X}\right)$ which is referred to as the variation intensity or the specific variation of the stationary random set $X$. Similarly, $\theta_{V_{u}}(X):=\theta_{V_{u}}\left(\mathbb{1}_{X}\right)$ is called the directional variation intensity or the specific directional variation in the direction $u$. Let $X$ be a stationary random set. The variogram $\nu_{X}$ of $X$ is the function $\nu_{X}: \mathbb{R}^{d} \rightarrow \mathbb{R}$ defined by $\nu_{X}(y)=\mathbb{P}(y \in X, 0 \notin X)$. Remark that since $X$ is a random set, $\left|\mathbb{1}_{X}(r u)-\mathbb{1}_{X}(0)\right| \in$ $\{0,1\}$, and thus, $\mathbb{E}\left(\left|\mathbb{1}_{X}(r u)-\mathbb{1}_{X}(0)\right|\right)=\mathbb{P}(r u \in X, 0 \notin X)+\mathbb{P}(r u \notin X, 0 \in X)=$ $2 \nu_{X}(r u)$. By Theorem 3, for all $u \in S^{d-1}$ the limit $\left(\nu_{X}^{u}\right)^{\prime}(0):=\lim _{r \rightarrow 0} \frac{1}{|r|} \nu_{X}(r u) \in$ $[0,+\infty]$ exists, and the specific directional variation $\theta_{V_{u}}(X)$ is given by

$$
\theta_{V_{u}}(X)=2\left(\nu_{X}^{u}\right)^{\prime}(0)=2 \lim _{r \rightarrow 0} \frac{1}{|r|} \mathbb{P}(r u \in X, 0 \notin X),
$$

showing that the specific directional variation is twice the directional derivative of the variogram at the origin [10]. Integrating over all directions, one obtains that

$$
\theta_{V}(X)=\frac{1}{\omega_{d-1}} \int_{S^{d-1}}\left(\nu_{X}^{u}\right)^{\prime}(0) \mathcal{H}^{d-1}(d u)
$$

This shows that Theorem 17 of [10] extends to jointly measurable stationary indicator functions that are not necessarily closed sets.

To finish this section, let us establish a mean corea formula for random excursion sets. First let us recall the coarea formula for deterministic functions. In what follows, $U$ is an open subset of $\mathbb{R}^{d}$. Recall that for any measurable set $A$, one defines the perimeter of $A$ in $U$ as the variation of the indicator function $\mathbb{1}_{A}$ in $U$, and one writes $\operatorname{Per}(A, U):=\left|D \mathbb{1}_{A}\right|(U)$. Similarly, one defines $V_{u}(A, U):=\left|D_{u} \mathbb{1}_{A}\right|(U)$ the directional variation of $A$ in $U$. If $f: U \rightarrow \mathbb{R}$ is a measurable function and $t \in \mathbb{R},\{f>t\}$ denotes the set $\{x \in U, f(x)>t\}$ and is called the (upper) level set of level $t$ of $f$. 
Proposition 5. (Coarea formula.) Let $f \in L^{1}(U)$. Then $f \in B V(U)$ if and only if the sets $\{f>t\}$ are of finite perimeter for $\mathcal{L}^{1}$-a.e. $t \in \mathbb{R}$ and the function $t \mapsto$ $\operatorname{Per}(\{f>t\}, U)$ is in $L^{1}(\mathbb{R})$, and in this case $|D f|(U)=\int_{-\infty}^{+\infty} \operatorname{Per}(\{f>t\}, U) d t$.

We refer to [2, p. 145] for the proof of the coarea formula. Let us mention that the coarea formula remains valid if the upper level sets are replaced by other level sets: $\{f \geq t\},\{f<t\}$ or $\{f \leq t\}$. Besides, a coarea formula also holds for directional variation: $\left|D_{u} f\right|(U)=\int_{-\infty}^{+\infty} D_{u}(\{f>t\}, U) d t, u \in S^{d-1}$. Using the coarea formula, one obtains a relation between the variation intensity $\Theta_{V_{u}}(f, U)=\mathbb{E}\left(\left|D_{u} f\right|(U)\right)$ of $f$ and the variation intensity of its level sets.

Proposition 6. (Coarea formula for total variation intensity.) Let $f$ be a r.f. a.s. in $L^{1}(U)$, and let $u \in S^{d-1}$. Then $f \in B V_{u}(U)$ a.s. with finite mean total directional variation $\Theta_{V_{u}}(f, U)$ if and only if for $\mathcal{L}^{1}$-a.e. $t \in \mathbb{R}$ its level sets $\{f>t\}$ have a.s. finite directional variation in $U$ in the direction $u$ and $t \mapsto \Theta_{V_{u}}(\{f>t\}, U)$ is in $L^{1}(\mathbb{R})$, and in this case $\Theta_{V_{u}}(f, U)=\int_{-\infty}^{+\infty} \Theta_{V_{u}}(\{f>t\}, U) d t$. Similarly, $f \in B V(U)$ a.s. with finite mean total variation if and only if for $\mathcal{L}^{1}$-a.e. $t \in \mathbb{R}$ its level sets $\{f>t\}$ have a.s. finite variation and $t \mapsto \Theta_{V}(\{f>t\}, U)$ is in $L^{1}(\mathbb{R})$, and in this case $\Theta_{V}(f, U)=\int_{-\infty}^{+\infty} \Theta_{V}(\{f>t\}, U) d t$.

Proof. The proof consists in applying Fubini and Lebesgue theorem. First, let us justify that the function $g: \Omega \times U \times \mathbb{R} \rightarrow\{0,1\}$ defined by $g(\omega, x, t)=\mathbb{1}_{\{f>t\}}(\omega, x)$ is measurable. Let $\left(t_{n}\right)_{n \in \mathbb{N}}$ be a dense sequence of $\mathbb{R}$, then one easily checks that

$$
g^{-1}(1)=\bigcup_{n \in \mathbb{N}}\left\{(\omega, x), f(\omega, x)>t_{n}\right\} \times\left(t_{n},+\infty\right),
$$

which is in the product $\sigma$-algebra $\mathcal{A} \otimes \mathcal{B}(U) \otimes \mathcal{B}(\mathbb{R})$ since $f$ is jointly measurable. Second, we have the following elementary identity

$$
|f(\omega, x+r u)-f(\omega, x)|=\int_{-\infty}^{+\infty}\left|\mathbb{1}_{\{f>t\}}(\omega, x+r u)-\mathbb{1}_{\{f>t\}}(\omega, x)\right| d t .
$$

Hence, by Fubini theorem

$\int_{U \ominus[0, r u]} \mathbb{E}(|f(x+r u)-f(x)|) d x=\int_{-\infty}^{+\infty} \int_{U \ominus[0, r u]} \mathbb{E}\left(\left|\mathbb{1}_{\{f>t\}}(x+r u)-\mathbb{1}_{\{f>t\}}(x)\right|\right) d x d t$.

Let us now suppose that for $\mathcal{L}^{1}$-a.e. $t \in \mathbb{R}$ the level sets $\{f>t\}$ have a.s. finite directional variation in the direction $u$ and that $t \mapsto \Theta_{V_{u}}(\{f>t\}, U)$ is in $L^{1}(\mathbb{R})$. Then, 
by Proposition $3, \lim _{r \rightarrow 0} \int_{U \ominus[0, r u]} \mathbb{E}\left(\left|\mathbb{1}_{\{f>t\}}(x ; r u)\right|\right) d x=\Theta_{V_{u}}(\{f>t\}, U)$ for $\mathcal{L}^{1}-$ a.e. $t$, and by Lemma $1, \int_{U \ominus[0, r u]} \mathbb{E}(|f(x ; r u)|) d x \leq \Theta_{V_{u}}(\{f>t\}, U) \in L^{1}(\mathbb{R})$. Hence Lebesgue theorem applies and $\lim _{r \rightarrow 0} \int_{U \ominus[0, r u]} \mathbb{E}(|f(x ; r u)|) d x=\int_{-\infty}^{+\infty} \Theta_{V_{u}}(\{f>t\}, U) d t<$ $+\infty$. By Proposition 3 one deduces that $f$ has a.s. bounded directional variation and that $\Theta_{V_{u}}(f, U)=\int_{-\infty}^{+\infty} \Theta_{V_{u}}(\{f>t\}, U) d t$. Let us now prove the converse implication. Suppose that $f \in B V_{u}(U)$ a.s. with $\Theta_{V_{u}}(f, U)<+\infty$. Then, by Fatou lemma and Fubini theorem

$\int_{-\infty}^{+\infty} \liminf _{r \rightarrow 0} \int_{U \ominus[0, r u]} \mathbb{E}\left(\left|\mathbb{1}_{\{f>t\}}(x ; r u)\right|\right) d x d t \leq \liminf _{r \rightarrow 0} \int_{U \ominus[0, r u]} \mathbb{E}(|f(x ; r u)|) d x=\Theta_{V_{u}}(f, U)<+\infty$.

In particular, for $\mathcal{L}^{1}$-a.e. $t \in \mathbb{R}, \liminf _{r \rightarrow 0} \int_{U \ominus[0, r u]}\left|\mathbb{1}_{\{f>t\}}(x ; r u)\right| d x<+\infty$ a.s. Hence, for $\mathcal{L}^{1}$-a.e. $t \in \mathbb{R}$, Proposition 3 ensures that $\{f>t\}$ has a.s. locally bounded variation in $U$ in the direction $u$, and that $\Theta_{V_{u}}(\{f>t\}, U)=\lim _{r \rightarrow 0} \int_{U \ominus[0, r u]} \mathbb{E}\left(\left|\mathbb{1}_{\{f>t\}}(x ; r u)\right|\right) d x$. Besides, the above inequality shows that $\int_{-\infty}^{+\infty} \Theta_{V_{u}}(\{f>t\}, U) \leq \Theta_{V_{u}}(f, U)<+\infty$, that is $t \mapsto \Theta_{V_{u}}(\{f>t\}, U) \in L^{1}(\mathbb{R})$. To finish, the case of non directional variation easily follows from the integral geometric formula of Proposition 1 and Fubini theorem.

Remark 6. Since the level sets $\{f>t\}, t \in \mathbb{R}$, of a stationary r.f. $f$ are stationary random sets, when dealing with stationary r.f. , Proposition 6 gives a similar results for variation intensities, that is,

$$
\theta_{V_{u}}(f)=\int_{-\infty}^{+\infty} \theta_{V_{u}}(\{f>t\}) d t \quad \text { and } \quad \theta_{V}(f)=\int_{-\infty}^{+\infty} \theta_{V}(\{f>t\}) d t .
$$

\section{Illustration: variation intensities of some classical random field models}

This section illustrates the different results of Section 4 and 5 by computing the variation intensities of various r.f. and random set models, namely Gaussian random fields, Gaussian excursion sets, Poisson shot noise of random sets, Boolean models, colored dead leaves r.f. , and colored tessellations. Most of the obtained formulas remains valid in the degenerate cases, and thus provides a characterization for the finiteness of the variation intensities of the considered r.f. or random set models.

\subsection{Gaussian random fields and Gaussian excursion sets}

A jointly measurable r.f. $f_{\mathrm{G}}$ is a stationary Gaussian r.f. with mean $\mu \in \mathbb{R}$ and covariance function $C: \mathbb{R}^{d} \rightarrow \mathbb{R}$ if for all $p \in \mathbb{N}, x_{1}, \ldots, x_{p} \in \mathbb{R}^{d}$, and $w_{1}, \ldots, w_{p} \in \mathbb{R}$ 
the r.v. $\sum_{i=1}^{p} w_{i} f_{\mathrm{G}}\left(x_{i}\right)$ is normal with mean $\sum_{i=1}^{p} w_{i} \mu$ and variance $\sum_{i, j=1}^{p} w_{i} w_{j} C\left(x_{j}-x_{i}\right)$. Let us mention that the measurability assumption is not restrictive since in what follows we will only consider stationary Gaussian r.f. whose covariance function is regular at the origin, and as soon as the covariance function $C$ is continuous in 0 , the r.f. is continuous in probability and thus it has a measurable version [13, p. 171]. For all $t \in \mathbb{R}$, we consider the random set $\left\{f_{\mathrm{G}}>t\right\}$, called Gaussian excursion set, and we denote by $\nu_{t}$ its variogram, that is the function defined for all $y \in \mathbb{R}^{d}$ by $\nu_{t}(y)=\mathbb{P}\left(f_{\mathrm{G}}(y)>t, f_{\mathrm{G}}(0) \leq t\right)$. As shown in [18, Proposition 16.1.1],

$$
\nu_{t}(y)=\frac{1}{\pi} \int_{0}^{\arcsin \left(\sqrt{\frac{C(0)-C(y)}{2 C(0)}}\right)} \exp \left(-\frac{t^{2}}{2 C(0)}\left(1+\tan ^{2} s\right)\right) d s .
$$

From this expression Lantuéjoul asserts that the excursion sets of $f_{\mathrm{G}}$ have finite "specific perimeter" if and only if $C(0)-C(y)$ is proportional to $|y|^{2}[18$, p. 207]. Our next proposition completes this observation in computing the expression of the specific variations of the Gaussian excursion sets $\left\{f_{\mathrm{G}}>t\right\}$, as well as the variation intensity of the Gaussian r.f. $f_{\mathrm{G}}$.

Proposition 7. (Variation intensity of stationary Gaussian r.f..) Let $f_{\mathrm{G}}$ be a stationary Gaussian r.f. with mean $\mu$ and covariance $C$, and let $u \in S^{d-1}$.

(i) (variation intensity) $f_{\mathrm{G}}$ has finite directional variation intensity $\theta_{V_{u}}\left(f_{\mathrm{G}}\right)$ in the direction $u$ if and only if the one-dimensional restriction of the covariance $C_{u}$ : $r \mapsto C(r u)$ is twice differentiable at 0 , and in this case, noting $C_{u}^{\prime \prime}(0)$ the second derivative in 0 of $C_{u}, \theta_{V_{u}}\left(f_{\mathrm{G}}\right)=\sqrt{\frac{-2 C_{u}^{\prime \prime}(0)}{\pi}}$. Consequently, $f_{\mathrm{G}}$ has finite variation intensity $\theta_{V}\left(f_{\mathrm{G}}\right)$ if and only if for all $u \in S^{d-1}$ the one-dimensional restrictions of the covariance $C_{u}: r \mapsto C(r u)$ are twice differentiable at 0 , and in this case, $\theta_{V}\left(f_{\mathrm{G}}\right)=\frac{1}{2 \omega_{d-1}} \int_{S^{d-1}} \sqrt{\frac{-2 C_{u}^{\prime \prime}(0)}{\pi}} \mathcal{H}^{d-1}(d u)$.

(ii) (variation intensity of excursion sets) For all $t \in \mathbb{R},\left\{f_{\mathrm{G}}>t\right\}$ has finite specific directional variation in the direction $u$ if and only if the one-dimensional restriction $C_{u}$ of the covariance is twice differentiable at 0 , and in this case,

$$
\theta_{V_{u}}\left(\left\{f_{\mathrm{G}}>t\right\}\right)=\sqrt{\frac{-2 C_{u}^{\prime \prime}(0)}{\pi}} \frac{1}{\sqrt{2 \pi C(0)}} \exp \left(-\frac{t^{2}}{2 C(0)}\right)=\theta_{V_{u}}\left(f_{\mathrm{G}}\right) p_{f_{\mathrm{G}}}(t),
$$


where $p_{f_{\mathrm{G}}}(t)=\frac{1}{\sqrt{2 \pi C(0)}} \exp \left(-\frac{t^{2}}{2 C(0)}\right)$ is the first-order density of the r.f. $f_{\mathrm{G}}$. Consequently, $\left\{f_{\mathrm{G}}>t\right\}$ has finite specific variation in the direction $u$ if and only if for all $u \in S^{d-1}$ the one-dimensional restrictions $C_{u}: r \mapsto C(r u)$ are twice differentiable at 0 , and in this case, $\theta_{V}\left(\left\{f_{\mathrm{G}}>t\right\}\right)=\theta_{V}\left(f_{\mathrm{G}}\right) p_{f_{\mathrm{G}}}(t)$.

Proof. This is a straightforward application of Theorem 3. For all $u \in S^{d-1}$ and $r \in \mathbb{R}, f_{\mathrm{G}}(r u)-f_{\mathrm{G}}(0)$ follows a normal distribution with mean 0 and variance $2(C(0)-C(r u))$, and thus $\mathbb{E}\left(\left|f_{\mathrm{G}}(r u)-f_{\mathrm{G}}(0)\right|\right)=\frac{2}{\sqrt{\pi}} \sqrt{C(0)-C(r u)}$. Hence, $\lim _{r \rightarrow 0} \mathbb{E}\left(\left|f_{\mathrm{G}}(0 ; r u)\right|\right)$ exists if and only if $\frac{C(0)-C(r u)}{r^{2}}$ admits a limit in 0 , that is if and only if $C_{u}: r \mapsto C(r u)$ is twice differentiable at 0 with $C_{u}^{\prime}(0)=0$. But since $C_{u}$ is even, if it is differentiable at 0 then necessarily $C_{u}^{\prime}(0)=0$. As for the expression of $\theta_{V_{u}}\left(f_{\mathrm{G}}\right)$, note that $\lim _{r \rightarrow 0} \frac{C(0)-C(r u)}{r^{2}}=-\frac{C_{u}^{\prime \prime}(0)}{2}$, hence by Theorem 3 $\theta_{V_{u}}\left(f_{\mathrm{G}}\right)=\lim _{r \rightarrow 0} \mathbb{E}\left(\left|f_{\mathrm{G}}(0 ; r u)\right|\right)=\sqrt{\frac{-2 C_{u}^{\prime \prime}(0)}{\pi}}$. The case of non directional variation follows by Formula (9).

Let us now consider excursion sets. By Formula (12), $\theta_{V_{u}}\left(\left\{f_{\mathrm{G}}>t\right\}\right)=2 \lim _{r \rightarrow 0} \frac{\nu_{t}(r u)}{|r|}$. The function $h: x \mapsto \frac{1}{\pi} \int_{0}^{\arcsin (x)} \exp \left(-\frac{t^{2}}{2 C(0)}\left(1+\tan ^{2} s\right)\right) d s$ is $\mathcal{C}^{1}$ at $x=0$ and

$$
h^{\prime}(0)=\arcsin ^{\prime}(0) \frac{1}{\pi} \exp \left(-\frac{t^{2}}{2 C(0)}\left(1+\tan ^{2}(\arcsin (0))\right)\right)=\frac{1}{\pi} \exp \left(-\frac{t^{2}}{2 C(0)}\right) .
$$

Hence, thanks to Formula (15) one deduces that

$\theta_{V_{u}}\left(\left\{f_{\mathrm{G}}>t\right\}\right)=2 \lim _{r \rightarrow 0} \frac{\nu_{t}(r u)}{|r|}=\frac{2}{\pi} \exp \left(-\frac{t^{2}}{2 C(0)}\right) \lim _{r \rightarrow 0} \sqrt{\frac{C(0)-C(r u)}{2 C(0) r^{2}}}=\frac{2}{\pi} \exp \left(-\frac{t^{2}}{2 C(0)}\right) \sqrt{\frac{-C_{u}^{\prime \prime}(0)}{4 C(0)}}$.

Remark 7. (Regularity of Gaussian r.f.) It is worth noticing that the necessary and sufficient condition for a stationary Gaussian r.f. to be of locally bounded variation implies a stronger regularity on the sample paths than just being of locally bounded variation. First, the differentiability at the origin of the covariance implies that there exists $\rho>0$ and $K>0$ such that for all $x \in B(0, \rho), C(0)-C(x) \leq K|x|$, and thus the sample paths of stationary Gaussian r.f. $f_{\mathrm{G}}$ are Hölder continuous for all exponents $s<\frac{1}{2}$ (see [6, Proposition 5.2] that summarizes results from [1]). In addition, according to [24], if the covariance function $C$ is twice differentiable at the origin in every direction, then the sample paths of a stationary Gaussian r.f. $f_{\mathrm{G}}$ are a.s. in the Sobolev space $W_{\text {loc }}^{1,2}\left(\mathbb{R}^{d}\right) \subset W_{\text {loc }}^{1,1}\left(\mathbb{R}^{d}\right)$. Hence by Proposition $7, \theta_{V}\left(f_{\mathrm{G}}\right)<+\infty$ implies that $f_{\mathrm{G}} \in W_{\mathrm{loc}}^{1,1}\left(\mathbb{R}^{d}\right)$ a.s., and consequently its variation $\left|D f_{\mathrm{G}}\right|$ is absolutely 
continuous with respect to the Lebesgue measure. Hence, if $f_{\mathrm{G}} \in B V_{\mathrm{loc}}\left(\mathbb{R}^{d}\right)$ a.s. and has a finite variation intensity, then it is a "smooth" function among the functions of locally bounded variation since its variation measure $D f_{\mathrm{G}}$ has neither a jump part nor a Cantor part (see [2, Section 3.9] for more details on the decomposition of the variation measure $D f$ of functions of bounded variation). This shows that the space of functions of locally bounded variation is not of interest for stationary Gaussian r.f.

Remark 8. (Regularity of Gaussian excursion sets.) For all $t \in \mathbb{R}$ we have

$$
\theta_{V_{u}}\left(\left\{f_{\mathrm{G}}>t\right\}\right)=\theta_{V_{u}}\left(f_{\mathrm{G}}\right) p_{f_{\mathrm{G}}}(t) \quad \text { and } \quad \theta_{V}\left(\left\{f_{\mathrm{G}}>t\right\}\right)=\theta_{V}\left(f_{\mathrm{G}}\right) p_{f_{\mathrm{G}}}(t)
$$

and one can check that the coarea formulas of Equation (14) hold. These formulas show that the regularity of a stationary Gaussian r.f. corresponds to the one of its level sets: a stationary Gaussian r.f. has finite variation intensity if and only if all its level sets have finite variation intensities if and only if at least one of its level sets has finite variation intensity. Let us also note that the formula $\theta_{V}\left(\left\{f_{\mathrm{G}}>t\right\}\right)=\theta_{V}\left(f_{\mathrm{G}}\right) p_{f_{\mathrm{G}}}(t)$ is in accordance with the "Rice formula for the expectation of the geometric measure of level set" derived by Azaïs and Wschebor [4, Theorem 6.8]. Indeed, under some smoothness assumption on the paths of $f_{\mathrm{G}}$, thanks to the stationarity of $f_{\mathrm{G}}$ one has [4, Theorem 6.8] $\mathbb{E}\left(\mathcal{H}^{d-1}\left(\left\{y, f_{\mathrm{G}}(y)=t\right\} \cap(0,1)^{d}\right)\right)=\mathbb{E}(\|\nabla f(0)\| \mid f(0)=t) p_{f_{\mathrm{G}}}(t)$. But since for a stationary Gaussian r.f. , $\nabla f(0)$ and $f(0)$ are independent, one gets

$\mathbb{E}\left(\mathcal{H}^{d-1}\left(\left\{y, f_{\mathrm{G}}(y)=t\right\} \cap(0,1)^{d}\right)\right)=\mathbb{E}(\|\nabla f(0)\|) p_{f_{\mathrm{G}}}(t)=\theta_{V}\left(f_{\mathrm{G}}\right) p_{f_{\mathrm{G}}}(t)=\theta_{V}\left(\left\{f_{\mathrm{G}}>t\right\}\right)$.

\subsection{Germ-grain models}

In this section we compute the variation intensities of several germ-grain models under very broad assumptions for the grain distribution. A germ-grain model defines a r.f. by combining a collection of colored random sets given as a marked Poisson process according to an interaction principle (addition, supremum, occultation,...). The random sets are called the grains while the random sets location are called the germs [29, 18]. Although most of the models evoked here could be defined using RAMS to define the grains (see also the recent paper of Rataj regarding random sets of finite perimeter [22]), we prefer to use the classical framework of RACS to simplify the presentation. 
6.2.1. Poisson shot noise Let us first consider the Poisson shot noise model that is obtained in summing random functions placed on the points of a Poisson process. More formally, the Poisson shot noise associated with the independently marked Poisson process $\Pi=\left\{\left(x_{j}, \kappa_{j}\right)\right\} \subset \mathbb{R}^{d} \times K$ and the impulse function $h: \mathbb{R}^{d} \times K \rightarrow \mathbb{R}$ is the r.f. $f_{\mathrm{SN}}$ defined by

$$
f_{\mathrm{SN}}(x)=\sum_{\left(x_{j}, \kappa_{j}\right) \in \Pi} h\left(x-x_{j}, \kappa_{j}\right)
$$

where $\Pi=\left\{\left(x_{j}, \kappa_{j}\right)\right\} \subset \mathbb{R}^{d} \times K$ is an independently marked Poisson process having intensity measure $\lambda \mathcal{L}^{d} \otimes P_{\kappa}, \lambda>0$ and $P_{\kappa}$ is the probability distribution of the marks (see e.g. [18]). The impulse function $h: \mathbb{R}^{d} \times K \rightarrow \mathbb{R}$ is supposed to be $\mathcal{L}^{d} \otimes P_{\kappa^{-}}$ integrable, which ensures that $f_{\mathrm{SN}}$ has finite expectation by Campbell theorem (see e.g. [17]). We first show that if the impulse function $h$ has a finite mean total variation then $f_{\mathrm{SN}}$ has locally bounded variation and its variation intensity is finite.

Proposition 8. (Bounds on the variation intensities of Poisson shot noises.) Let $u \in S^{d-1}$ and suppose that $h(\cdot, \kappa) \in B V_{u}\left(\mathbb{R}^{d}\right) P_{\kappa}$-a.s. with $\mathbb{E}\left(\left|D_{u} h(\cdot, \kappa)\right|\left(\mathbb{R}^{d}\right)\right)<+\infty$. Then the shot noise $f_{\mathrm{SN}}$ has locally bounded directional variation in the direction $u$ and $\theta_{V_{u}}\left(f_{\mathrm{SN}}\right) \leq \lambda \mathbb{E}\left(\left|D_{u} h(\cdot, \kappa)\right|\left(\mathbb{R}^{d}\right)\right)$. Consequently, if $h(\cdot, \kappa) \in B V\left(\mathbb{R}^{d}\right) P_{\kappa}$-a.s. and $\mathbb{E}\left(|D h(\cdot, \kappa)|\left(\mathbb{R}^{d}\right)\right)<+\infty$ then $f_{\mathrm{SN}}$ has locally bounded variation and $\theta_{V}\left(f_{\mathrm{SN}}\right) \leq$ $\lambda \mathbb{E}\left(|D h(\cdot, \kappa)|\left(\mathbb{R}^{d}\right)\right)$.

Proof. Let $u \in S^{d-1}$ and $r \in \mathbb{R}$. Using triangular inequality and Campbell theorem (see e.g. [17]),

$$
\begin{aligned}
\mathbb{E}\left(\left|f_{\mathrm{SN}}(r u)-f_{\mathrm{SN}}(0)\right|\right) & \leq \mathbb{E}\left(\sum_{\left(x_{j}, \kappa_{j}\right) \in \Pi}\left|h\left(r u-x_{j}, \kappa_{j}\right)-h\left(-x_{j}, \kappa_{j}\right)\right|\right) \\
& \leq \lambda \int_{\mathbb{R}^{d} \times K}|h(r u-x, \kappa)-h(-x, \kappa)| d x P_{\kappa}(d \kappa)
\end{aligned}
$$

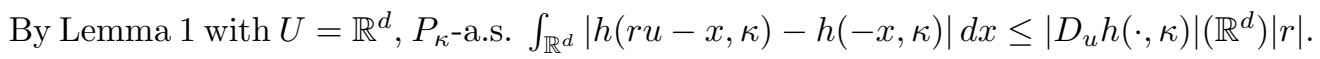
Hence $\mathbb{E}\left(\left|f_{\mathrm{SN}}(r u)-f_{\mathrm{SN}}(0)\right|\right) \leq \lambda \mathbb{E}\left(\left|D_{u} h(\cdot, \kappa)\right|\left(\mathbb{R}^{d}\right)\right)|r|$, that is $f_{\mathrm{SN}}$ is directionally Lipschitz in mean. By Proposition 4 , one concludes that $f_{\mathrm{SN}}$ is a.s. in $B V_{u, \text { loc }}\left(\mathbb{R}^{d}\right)$ and $\theta_{V_{u}}\left(f_{\mathrm{SN}}\right) \leq \lambda \mathbb{E}\left(\left|D_{u} h(\cdot, \kappa)\right|\left(\mathbb{R}^{d}\right)\right)$. The upper bound on $\theta_{V}\left(f_{\mathrm{SN}}\right)$ is obtained in integrating over all directions $u \in S^{d-1}$. 
Proposition 8 simply establishes that the mean variation of the sum is lower than the sum of the mean variations. It only gives an upper bound on the variation intensity of the Poisson shot noise. Biermé and Desolneux [5] recently studied the mean perimeter of excursion sets of Poisson shot noise models within the framework of functions of bounded variation with more specific techniques. Under the hypothesis that the functions $h(\cdot, \kappa)$ have no Cantor part, they obtained an expression for the mean variation intensity of Poisson shot noises [5, Theorem 3] which is the sum of two terms: one term for the approximate differential of $f_{\mathrm{SN}}[2]$ and one from the jump part of $f_{\mathrm{SN}}$. This second term is explicit and results from the observation that the jump part of $f_{\mathrm{SN}}$ is the sum of the jump parts of the derivatives $D h\left(\cdot-x_{j}, \kappa_{j}\right)$. Consequently, when the functions $h$ are indicator functions of sets of finite perimeter, the upper bound of Proposition 8 is reached, and thus Proposition 8 cannot be improved in general. The following proposition sums up this observation.

Proposition 9. (Variation of shot noises of random indicator functions.) Consider a shot noise of the form

$$
f_{\mathrm{SN}}(x)=\sum_{\left(x_{j}, a_{j}, X_{j}\right) \in \Pi} a_{j} \mathbb{1}\left(x \in x_{j}+X_{j}\right)
$$

where the Poisson process $\Pi_{\lambda}$ has intensity measure $\lambda \mathcal{L}^{d} \otimes P_{X} \otimes P_{a}, \lambda \geq 0, P_{X} a$ probability distribution over the set $\mathcal{F}$ of closed subsets of $\mathbb{R}^{d}$, and $P_{a} \in L^{1}(\Omega)$. Suppose that the RACS $X \sim P_{X}$ has finite mean Lebesgue measure and finite perimeter. Then $f_{\mathrm{SN}}$ has a.s. bounded variation and

$$
\theta_{V}(f)=\lambda \mathbb{E}(|a|) \mathbb{E}(\operatorname{Per}(X)) \quad \text { and } \quad \theta_{V_{u}}(f)=\lambda \mathbb{E}(|a|) \mathbb{E}\left(V_{u}(X)\right), \quad u \in S^{d-1}
$$

Proof. The formula $\theta_{V}(f)=\lambda \mathbb{E}(|a|) \mathbb{E}(\operatorname{Per}(X))$ is given by [5, Theorem 3]. By Proposition 8, this implies that $\theta_{V_{u}}(f)=\lambda \mathbb{E}(|a|) \mathbb{E}\left(V_{u}(X)\right)$ for $\mathcal{H}^{d-1}$-almost all $u \in$ $S^{d-1}$. We conclude that it is true for all $u$ using a continuity argument (see Remark 1 ).

6.2.2. Boolean models We now turn to the computation of the variation intensities of Boolean models. Recall that the homogeneous Boolean random set with intensity $\lambda$ and grain distribution $P_{X}$ is the stationary random closed sets (RACS) $Z_{\mathrm{B}}$ defined by $Z_{\mathrm{B}}=$ $\bigcup_{j \in \mathbb{N}} x_{j}+X_{j}$ where $\left\{\left(x_{j}, X_{j}\right)\right\}$ is an independently marked stationary Poisson process 
in the space $\mathbb{R}^{d} \times \mathcal{F}$ having intensity measure $\lambda \mathcal{L}^{d} \otimes P_{X}, \lambda \geq 0$ (see e.g. [29, 18, 25]). Starting from the well-known expression of the variogram of the Boolean set $Z_{\mathrm{B}}$, it has been shown in [10] using Formula (12) that

$$
\begin{gathered}
\theta_{V_{u}}\left(Z_{\mathrm{B}}\right)=\lambda \mathbb{E}\left(V_{u}(X)\right) \exp \left(-\lambda \mathbb{E}\left(\mathcal{L}^{d}(X)\right)\right), \quad u \in S^{d-1}, \\
\theta_{V}\left(Z_{\mathrm{B}}\right)=\lambda \mathbb{E}(\operatorname{Per}(X)) \exp \left(-\lambda \mathbb{E}\left(\mathcal{L}^{d}(X)\right)\right) .
\end{gathered}
$$

As discussed in [10], let us recall that Equation (16) is valid for any grain distribution $P_{X}$ and that it generalizes known results for Boolean models with convex grains [25, p. 386]. Similar generalizations involving intensity of surface measures deriving from Steiner formula have recently been established $[14,31]$, under some technical hypotheses on the RACS $X$. The above formulas are similar but not identical since the outer Minkowski content of a set differs from its (variational) perimeter [30].

Several r.f. models can be considered as generalizations of Boolean random sets [27]. Here we consider a simple example called random Boolean islands. Let $\Phi=\left\{\left(x_{j}, X_{j}, a_{j}\right)\right\}$ be an independently marked Poisson process taking values in $\mathbb{R}^{d} \times \mathcal{F} \times[0,+\infty)$ and having intensity measure $\lambda \mathcal{L}^{d} \otimes P_{X} \otimes P_{a}, \lambda \geq 0$. We define the Boolean random field $f_{\mathrm{B}}$ associated to this process by $f_{\mathrm{B}}(y)=\sup \left(\{0\} \cup\left\{a_{j}, y \in x_{j}+X_{j}\right\}\right)$. Remark that with this model, the colored random sets are superimposed according to a hierarchy: the lighter sets are placed above the darker ones. Note that if $a_{j}=1$ a.s., then $f_{\mathrm{B}}$ is the indicator function of the Boolean random set $Z_{\mathrm{B}}$. More generally, the upper level sets of $f_{\mathrm{B}}$ are Boolean random sets: indeed, for all $t \geq 0,\left\{y, f_{\mathrm{B}}(y)>t\right\}$ is the Boolean model associated with the Poisson process $\sum_{\Phi} \mathbb{1}\left(a_{j}>t\right) \delta_{x_{j}, X_{j}}$. Relying on this observation, one deduces an expression of the variation intensities of Boolean r.f. using the coarea formula for variation intensities.

Proposition 10. (Variation intensities of Boolean random fields.) Let $f_{\mathrm{B}}$ be the Boolean r.f. with Poisson intensity $\lambda$, grain distribution $P_{X}$, and gray-level distribution $P_{a}$. Let $X$ denote a RACS with distribution $P_{X}$ and let a denote a r.v. with distribution $P_{a}$. Then,

$$
\begin{gathered}
\theta_{V_{u}}\left(f_{\mathrm{B}}\right)=\lambda \mathbb{E}\left(V_{u}(A)\right) \int_{0}^{+\infty} P_{a}(\{a>t\}) \exp \left(-\lambda \mathbb{E}\left(\mathcal{L}^{d}(X)\right) P_{a}(\{a>t\})\right) d t, \quad u \in S^{d-1}, \\
\theta_{V}\left(f_{\mathrm{B}}\right)=\lambda \mathbb{E}(\operatorname{Per}(A)) \int_{0}^{+\infty} P_{a}(\{a>t\}) \exp \left(-\lambda \mathbb{E}\left(\mathcal{L}^{d}(X)\right) P_{a}(\{a>t\})\right) d t .
\end{gathered}
$$


Proof. $\left\{y, f_{\mathrm{B}}(y)>t\right\}$ is the Boolean model associated with the Poisson process $\sum_{\Phi} \mathbb{1}\left(a_{j}>t\right) \delta_{x_{j}, X_{j}}$. This Poisson process has grain distribution $P_{X}$ and intensity $\lambda P_{a}(\{a>t\})$. Hence $\theta_{V_{u}}\left(\left\{f_{\mathrm{B}}>t\right\}\right)=\lambda \mathbb{E}\left(V_{u}(A)\right) P_{a}(\{a>t\}) \exp \left(-\lambda \mathbb{E}\left(\mathcal{L}^{d}(X)\right) P_{a}(\{a>t\})\right)$. The expressions for $\theta_{V_{u}}\left(f_{\mathrm{B}}\right)$ and $\theta_{V}\left(f_{\mathrm{B}}\right)$ are obtained using the coarea formula for variation intensities (see Equation (14)).

6.2.3. Colored dead leaves model The dead leaves model $[9,16,7]$, also initially introduced by Matheron, is a germ-grain model where the interaction rule is occultation, that is where the grains $x_{j}+X_{j}$ hide each other. For this germ-grain model the grains are chronologically ordered by a time $t_{j} \in(-\infty, 0)$, called falling time. The leaves are the points of the Poisson process $\Phi=\left\{\left(t_{j}, x_{j}, X_{j}, a_{j}\right)\right\} \subset(-\infty, 0) \times \mathbb{R}^{d} \times \mathcal{F} \times \mathbb{R}$ with intensity measure $\mathcal{L}^{1} \otimes \mathcal{L}_{d} \otimes P_{X} \otimes P_{a}$. For each leaf $\left(t_{j}, x_{j}, X_{j}, a_{j}\right)$, the random set $x_{j}+X_{j}$ associated with the random color $a_{j}$ is partially or totally hidden by its subsequent leaves, that is the leaves which fall after $t=t_{j}$. In the end, at time $t=0$ the only remaining part of $x_{j}+X_{j}$ is the visible part $V_{j}$, that is the set

$$
V_{j}=\left(x_{j}+X_{j}\right) \backslash\left(\bigcup_{\left(t_{k}, x_{k}, X_{k}, a_{k}\right) \in \Phi, t_{k}>t_{j}} x_{k}+X_{k}\right) .
$$

As soon as $\mathbb{E}\left(\mathcal{L}^{d}(X)\right)>0$, the whole Euclidean space $\mathbb{R}^{d}$ is covered by the random sets $x_{j}+X_{j}$, and consequently each point $y \in \mathbb{R}^{d}$ belongs to a unique visible part. The colored dead leaves r.f. $f_{\mathrm{CDL}}$ is the r.f. defined in assigning to each $y \in \mathbb{R}^{d}$ the color $a_{j}$ of the unique visible part $V_{j}$ such that $y \in V_{j}$. More formally, $f_{\mathrm{CDL}}$ is defined by

$$
f_{\mathrm{CDL}}(y)=\sum_{\left(t_{j}, x_{j}, X_{j}, a_{j}\right) \in \Phi} a_{j} \mathbb{1}\left(y \in V_{j}\right)
$$

but note that for each point $y$ the above sum has only one non null term. Even though occultation between objects is also observable with Boolean r.f. , colored dead leaves r.f. vary from this first model: here the ordering of the objects is not related to their gray-level, and the whole domain is by construction fully covered by objects.

The next proposition gives the variation intensities of this r.f. model. Relying on Theorem 3, it consists in computing $\mathbb{E}\left(\left|f_{\mathrm{CDL}}(r u)-f_{\mathrm{CDL}}(0)\right|\right)$. To do so one should

Our definition of the visible parts $V_{j}$ is slightly different from the one of [7]. This is because we do not enforce the visible parts to be closed sets. 
consider the set of leaves that cover (or hit) the two points 0 and $r u$ (or both). Let us consider the restriction $\Phi^{\{0, r u\}}$ of the leaves of $\Phi$ which hit the set $\{0, r u\}$, that is

$$
\Phi^{\{0, r u\}}=\left\{\left(t_{j}, x_{j}, X_{j}, a_{j}\right) \in \Phi, x_{j}+X_{j} \cap\{0, r u\} \neq \emptyset\right\} .
$$

According to [11, Proposition 5], $\Phi^{\{0, r u\}}$ is an independently marked Poisson process with ground process $\left\{t_{j},\left(t_{j}, x_{j}, X_{j}, a_{j}\right) \in \Phi^{\{0, r u\}}\right\}$ of intensity $2 \gamma_{X}(0)-\gamma_{X}(r u)$ (where $\gamma_{X}$ denotes the mean geometric covariogram of $\left.X\right)$ and marks $\left(x_{j}, X_{j}, a_{j}\right)$. The marks $a_{j}$ are i.i.d. with distribution $P_{a}$, and are independent of $\left(x_{j}, X_{j}\right)$. As for the distribution of the marks $\left(x_{j}, X_{j}\right)$ we are only interested in the following probabilities. $\mathbb{P}\left(\{0, r u\} \subset x_{j}+X_{j}\right)=\frac{\mathcal{L}^{d} \otimes P_{X}(\{(x, X),\{0, r u\} \subset x+X\})}{\mathcal{L}^{d} \otimes P_{X}(\{(x, X),\{0, r u\} \cap x+X \neq \emptyset\})}=\frac{\gamma_{X}(r u)}{2 \gamma_{X}(0)-\gamma_{X}(r u)}$. and, by symmetry and complementarity, $\mathbb{P}\left(0 \in x_{j}+X_{j}\right.$ and $\left.r u \notin x_{j}+X_{j}\right)=\mathbb{P}\left(r u \in x_{j}+X_{j}\right.$ and $\left.0 \notin x_{j}+X_{j}\right)=\frac{\gamma_{X}(0)-\gamma_{X}(r u)}{2 \gamma_{X}(0)-\gamma_{X}(r u)}$. In addition, if one denotes by $\left(t_{0}, x_{0}, X_{0}, a_{0}\right)$ the last leaf of $\Phi^{\{0, r u\}}$, that is, the leaf such that $t_{0}=\sup \left\{t_{j},\left(t_{j}, x_{j}, X_{j}, a_{j}\right) \in \Phi^{\{0, r u\}}\right\}$, then $\left(x_{0}, X_{0}, a_{0}\right)$ has the same distribution as any mark $\left(x_{j}, X_{j}, a_{j}\right)$ of $\Phi^{\{0, r u\}}$, the shifted Poisson process $\Phi_{t_{0}}=$ $\left\{\left(t-t_{0}, x, X, a\right),(t, x, X, a) \in \Phi\right.$ and $\left.t<t_{0}\right\}$ has the same distribution as $\Phi$, and $\left(x_{0}, X_{0}, a_{0}\right)$ and $\Phi_{t_{0}}$ are independent.

Proposition 11. (Variation intensities of the colored dead leaves r.f..) Suppose that $0<\mathbb{E}\left(\mathcal{L}^{d}(X)\right)<+\infty$ and that $a \in L^{1}(\Omega)$. Let $a_{1}$ and $a_{2}$ be two independent r.v. with distribution $P_{a}$. Then for all $u \in S^{d-1}$,

$$
\theta_{V_{u}}\left(f_{\mathrm{CDL}}\right)=\mathbb{E}\left(\left|a_{1}-a_{2}\right|\right) \frac{\mathbb{E}\left(V_{u}(X)\right)}{\mathbb{E}\left(\mathcal{L}^{d}(X)\right)}, \quad \text { and } \quad \theta_{V}\left(f_{\mathrm{CDL}}\right)=\mathbb{E}\left(\left|a_{1}-a_{2}\right|\right) \frac{\mathbb{E}(\operatorname{Per}(X))}{\mathbb{E}\left(\mathcal{L}^{d}(X)\right)}
$$

Proof. Let us first compute the expectation $\mathbb{E}\left(\left|f_{\mathrm{CDL}}(r u)-f_{\mathrm{CDL}}(0)\right|\right)$ for $r \in \mathbb{R}$ and $u \in S^{d-1}$. If the points $r u$ and 0 are in the same visible part $V_{j}$, then $f_{\mathrm{CDL}}(r u)=$ $f_{\mathrm{CDL}}(0)$. Otherwise, if $r u$ and 0 are in different visible parts, then both $f_{\mathrm{CDL}}(r u)$ and $f_{\mathrm{CDL}}(0)$ have distribution $P_{a}$ and they are independent. Hence,

$\mathbb{E}\left(\left|f_{\mathrm{CDL}}(r u)-f_{\mathrm{CDL}}(0)\right|\right)=\mathbb{E}\left(\left|a_{1}-a_{2}\right|\right) \mathbb{P}(\{r u$ and 0 belong to different visible parts $\})$.

Now, $r u$ and 0 belong to different visible parts if the last leaf covering either $r u$ or 0 
does not cover both points. Noting $x_{0}+X_{0}$ the last leaf of $\Phi^{\{0, r u\}}$, we have

$$
\begin{aligned}
& \mathbb{P}(\{r u \text { and } 0 \text { belong to different visible parts }\}) \\
& =\mathbb{P}\left(0 \in x_{0}+X_{0} \text { and } r u \notin x_{0}+X_{0}\right)+\mathbb{P}\left(r u \in x_{0}+X_{0} \text { and } 0 \notin x_{0}+X_{0}\right) \\
& =2 \frac{\gamma_{X}(0)-\gamma_{X}(r u)}{2 \gamma_{X}(0)-\gamma_{X}(r u)} .
\end{aligned}
$$

Hence, by Theorem 3 and Equation (11)

$$
\begin{aligned}
\theta_{V_{u}}\left(f_{\mathrm{CDL}}\right)=\lim _{r \rightarrow 0} \mathbb{E}\left(\left|f_{\mathrm{CDL}}(0 ; r u)\right|\right) & =\mathbb{E}\left(\left|a_{1}-a_{2}\right|\right) \lim _{r \rightarrow 0} 2 \frac{\gamma_{X}(0)-\gamma_{X}(r u)}{|r|} \frac{1}{2 \gamma_{X}(0)-\gamma_{X}(r u)} \\
& =\mathbb{E}\left(\left|a_{1}-a_{2}\right|\right) \frac{\mathbb{E}\left(V_{u}(X)\right)}{\mathbb{E}\left(\mathcal{L}^{d}(X)\right)} .
\end{aligned}
$$

Integrating over all directions gives the expression of $\theta_{V}\left(f_{\mathrm{CDL}}\right)$.

Remark 9. The expression of the variation intensity $\theta_{V}\left(f_{\mathrm{CDL}}\right)=\mathbb{E}\left(\left|a_{1}-a_{2}\right|\right) \frac{\mathbb{E}(\operatorname{Per}(X))}{\mathbb{E}\left(\mathcal{L}^{d}(X)\right)}$ is in accordance with our expectation: Indeed, $\mathbb{E}\left(\left|a_{1}-a_{2}\right|\right)$ is the mean contrast between two distinct visible parts, whereas the ratio $\frac{\mathbb{E}(\operatorname{Per}(X))}{\mathbb{E}\left(\mathcal{L}^{d}(X)\right)}$ is known to be the mean length of cell boundary per unit area when the RACS are random polygons [9]. Let us also mention that the variation intensity of the transparent dead leaves random field defined in [11] can be computed using the same technique and that it takes the same form with a different contrast term depending on the degree of transparency.

6.2.4. Colored tessellations A colored tessellation is the r.f. obtained in assigning a random color to each cell of a random partition of the plane. The interaction principle which is at work for colored tessellations is arguably juxtaposition.

A (random) tessellation is a random partition $\bigcup_{j} C_{j}=\mathbb{R}^{d}$ of the Euclidean space $\mathbb{R}^{d}$, the sets $C_{j}$ being called cells of the tessellation. Even though random tessellations have been widely studied, there lacks a general acknowledged definition. This is principally because most studied tessellation models only involve convex cells. Nevertheless, tessellations can be constituted of non convex (and even non connected) cells, such as the tessellation corresponding to the dead leaves model defined in [7]. Following [28, 7], we consider a quite general definition: A (random) tessellation is a point process $T=\sum_{j} \delta_{C_{j}}$ taking values in the set $\mathcal{K}^{\prime}$ of non empty compact sets and which satisfies

\footnotetext{
Up to our slightly different definition of the visible parts, this probability is also given by the general Formula (12) of [7].
} 
the following additional properties: 1) For all compact sets $K$, the number of sets $C_{i}$ intersecting $K$ is finite; 2) For all $j \neq k$, $\left.\operatorname{int} C_{j} \cap \operatorname{int} C_{k} \neq \emptyset ; 3\right) \bigcup_{j} C_{k}=\mathbb{R}^{d}$;4) For all $j, \mathcal{L}^{d}\left(\partial C_{j}\right)=0$. With these conditions, a.e. point $x \in \mathbb{R}^{d}$ belongs to a unique cell $C_{j}$. We will only consider stationary tessellations, that is tessellations such that for all $x \in \mathbb{R}^{d}, \sum_{j} \delta_{x+C_{j}} \stackrel{\mathrm{d}}{=} \sum_{j} \delta_{C_{j}}$. Thanks to the stationarity, for these tessellations every point $x \in \mathbb{R}^{d}$ a.s. belongs to a unique cell $C_{j}$.

Given a stationary tessellation $T=\sum_{j} \delta_{C_{j}}$ one defines a stationary r.f. $f_{T}$ by associating a random intensity $a_{j} \in \mathbb{R}$ to each cell $C_{j}$. The real r.v. $a_{j}$ are i.i.d. with common distribution $P_{a}$. More formally, the colored tessellation $T_{c}$ associated to the tessellation $T$ and with color distribution $P_{a}$ is the independently marked point process $T_{c}=\sum_{j} \delta_{\left(C_{j}, a_{j}\right)}$, where the marks $a_{j}$ have common distribution $P_{a}$. If $\mu$ denotes the intensity measure of the point process $\sum_{j} \delta_{C_{j}}$, then $T_{c}=\sum_{j} \delta_{\left(C_{j}, a_{j}\right)}$ has intensity measure $\mu \otimes P_{a}$. Its associated r.f. $f_{T}$ is defined as follows: $f_{T}(x)=a_{j}$ where $a_{j}$ is the color of the a.s. unique cell $C_{j}$ containing $x$. Note that $f_{T}$ can also be defined as a sum over the marked point process $f_{T}(x)=\sum_{j} a_{j} \mathbb{1}\left(x \in C_{j}\right)$.

Before establishing the expression of the variation intensities of $f_{T}$ we need to introduce the fundamental notion of typical cell. First, one interprets a stationary tessellation as a point process in $\mathbb{R}^{d}$ marked with random sets by introducing a centroid map. Recall that $\mathcal{K}^{\prime}$ denotes the set of non empty compact sets of $\mathbb{R}^{d}$. A centroid map is a measurable application $z: \mathcal{K}^{\prime} \mapsto \mathbb{R}^{d}$ such that $z(x+C)=x+z(C)$. Second, given a centroid map $z$, any stationary tessellation $T=\sum_{j} \delta_{C_{j}}$ is decomposed into the stationary marked point process $\sum_{j} \delta_{\left(z\left(C_{j}\right), C_{j}-z\left(C_{j}\right)\right)}$. According to [25, Section 4.1], one deduces that for any stationary tessellation $T$ there exists a distribution $Q$ over $\mathcal{K}_{0}^{\prime}=\left\{K \in \mathcal{K}^{\prime}, z(K)=0\right\}$ such that for all measurable functions $f: \mathcal{K}^{\prime} \mapsto \mathbb{R}+$,

$$
\mathbb{E}\left(\sum_{j} f\left(C_{j}\right)\right)=\frac{1}{\mathbb{E}\left(\mathcal{L}^{d}(\mathcal{C})\right)} \int_{\mathcal{K}_{0}} \int_{\mathbb{R}^{d}} f(x+K) d x Q(d K),
$$

where, by definition, $Q$ is the distribution of the typical cell of $T$ denoted by $\mathcal{C} \sim$ $Q$. The key result to compute the variation intensity of randomly colored stationary tessellations is the following proposition ${ }^{\dagger}$.

\footnotetext{
$\dagger$ Proposition 12 is stated without proof in [18]. The proof reproduced below was personally communicated to the author by Pr. Pierre Calka.
} 
Proposition 12. (Stationary tessellations and mean covariogram.) Let $T=\sum_{j} \delta_{C_{j}}$ be a stationary tessellation and let $\gamma_{\mathcal{C}}: h \mapsto \mathbb{E}(\mathcal{C} \cap(h+\mathcal{C}))$ be the mean covariogram of its typical cell $\mathcal{C}$. Then for all $h \in \mathbb{R}^{d}, \mathbb{P}(\{0$ and $h$ belong to the same cell $\})=\frac{\gamma_{\mathcal{C}}(h)}{\gamma_{\mathcal{C}}(0)}$.

Proof. Denote $\rho(h)=\mathbb{P}(\{0$ and $h$ belong to the same cell $\})$. First, by stationarity $\rho(h)=\mathbb{P}(\{-h$ and 0 belong to the same cell $\})$. Second, remark that for all $C \in \mathcal{K}$, $\{0,-h\} \subset C \Longleftrightarrow 0 \in C \cap(h+C)$. Hence applying Formula (17) with $K \mapsto$ $\mathbb{1}(0 \in K \cap(h+K))$,

$$
\begin{aligned}
\rho(h) & =\mathbb{E}\left(\sum_{j} \mathbb{1}\left(0 \in C_{j} \cap\left(h+C_{j}\right)\right)\right) \\
& =\frac{1}{\mathbb{E}\left(\mathcal{L}^{d}(\mathcal{C})\right)} \int_{\mathcal{K}_{0}} \int_{\mathbb{R}^{d}} \mathbb{1}(0 \in(x+K) \cap(h+x+K)) d x Q(d K) \\
& =\frac{1}{\gamma_{\mathcal{C}}(0)} \int_{\mathcal{K}_{0}} \int_{\mathbb{R}^{d}} \mathbb{1}(-x \in K \cap(h+K)) d x Q(d K) \\
& =\frac{1}{\gamma_{\mathcal{C}}(0)} \int_{\mathcal{K}_{0}} \mathcal{L}^{d}(K \cap(h+K)) Q(d K)=\frac{\gamma_{\mathcal{C}}(h)}{\gamma_{\mathcal{C}}(0)} .
\end{aligned}
$$

Proposition 13. (Variation intensities of colored tessellations.) Let $T_{c}=\sum_{j} \delta_{\left(C_{j}, a_{j}\right)}$ be a randomly colored stationary tessellation, let $f_{T}$ be its associated stationary r.f. , and denote by $\mathcal{C}$ the typical cell of $T$. Let $a_{1}$ and $a_{2}$ be i.d.d. r.v. with distribution $P_{a}$. For all $u \in S^{d-1}, \theta_{V_{u}}\left(f_{T}\right)=\mathbb{E}\left(\left|a_{1}-a_{2}\right|\right) \frac{1}{2} \frac{\mathbb{E}\left(V_{u}(\mathcal{C})\right)}{\mathbb{E}\left(\mathcal{L}^{d}(\mathcal{C})\right)}$, and $\theta_{V}\left(f_{T}\right)=$ $\mathbb{E}\left(\left|a_{1}-a_{2}\right|\right) \frac{1}{2} \frac{\mathbb{E}(\operatorname{Per}(\mathcal{C}))}{\mathbb{E}\left(\mathcal{L}^{d}(\mathcal{C})\right)}$.

Proof. Let us compute $\mathbb{E}\left(\left|f_{T}(r u)-f_{T}(0)\right|\right)$. We have $\left|f_{T}(r u)-f_{T}(0)\right|=\left|a_{k}-a_{j}\right|$ if $0 \in C_{j}$ and $r u \in C_{k}$ with $j \neq k$, and 0 if 0 and $r u$ belong to the same cell. By Proposition 12 , and since for $j \neq k, a_{j}$ and $a_{k}$ are independent, $\mathbb{E}\left(\left|f_{T}(r u)-f_{T}(0)\right|\right)=$ $\mathbb{E}\left(\left|a_{1}-a_{2}\right|\right) \mathbb{P}(\{0$ and $r u$ are in different cells $\})=\mathbb{E}\left(\left|a_{1}-a_{2}\right|\right) \frac{\gamma_{\mathcal{C}}(0)-\gamma_{\mathcal{C}}(r u)}{\gamma_{\mathcal{C}}(0)}$. By Formula (11) applied with $X=\mathcal{C}$ and Theorem $3, \theta_{V_{u}}\left(f_{T}\right)=\mathbb{E}\left(\left|a_{1}-a_{2}\right|\right) \frac{1}{2} \frac{\mathbb{E}\left(V_{u}(\mathcal{C})\right)}{\mathbb{E}\left(\mathcal{L}^{d}(\mathcal{C})\right)}$. Integrating over all directions one obtains the expression of $\theta_{V}\left(f_{T}\right)$.

Observe that the formula $\theta_{V}\left(f_{T}\right)=\mathbb{E}\left(\left|a_{1}-a_{2}\right|\right) \frac{1}{2} \frac{\mathbb{E}(\operatorname{Per}(\mathcal{C}))}{\mathbb{E}\left(\mathcal{L}^{d}(\mathcal{C})\right)}$ is in accordance with our expectation: Indeed, $\mathbb{E}\left(\left|a_{1}-a_{2}\right|\right)$ is the mean contrast between two adjacent cells whereas $\frac{1}{2} \frac{\mathbb{E}(\operatorname{Per}(\mathcal{C}))}{\mathbb{E}\left(\mathcal{L}^{d}(\mathcal{C})\right)}$ is known to be the mean length of tessellations boundary per unit area [25, Section 10.1]. 


\section{Acknowledgements}

The author is grateful to Pr. Pierre Calka for his help for establishing the proof of Proposition 12, to Pr. Yann Gousseau for his various comments that contributed to improve a preliminary version of this paper, as well as to the reviewers for their relevant suggestions.

\section{References}

[1] Adler, R. (1981). The Geometry of Random Fields. Wiley Series in Probability and Mathematical Statistics. John Wiley \& Sons, Ltd., Chichester.

[2] Ambrosio, L., Fusco, N. and Pallara, D. (2000). Functions of Bounded Variation and Free Discontinuity Problems. Oxford mathematical monographs. The Clarendon Press, Oxford University Press, New York.

[3] Aubert, G. and Kornprobst, P. (2006). Mathematical Problems in Image Processing second ed. vol. 147 of Applied Mathematical Sciences. Springer-Verlag, New York.

[4] Azaïs, J.-M. and Wschebor, M. (2009). Level Sets and Extrema of Random Processes and Fields. John Wiley \& Sons, Inc., Hoboken, NJ.

[5] Biermé, H. And Desolneux, A. (2015). On the perimeter of excursion sets of shot noise random fields. Ann. Prob.. to appear.

[6] Biermé, H., Meerschaert, M. M. and Scheffler, H.-P. (2007). Operator scaling stable random fields. Stochastic Processes and their Applications 117, 312 -332 .

[7] Bordenave, C., Gousseau, Y. and Roueff, F. (2006). The dead leaves model: a general tessellation modeling occlusion. Adv. Appl. Probab. 38, 31-46.

[8] Chlebík, M. (1997). On variation of sets. preprint 44. Max-Planck-Institut für Mathematik in den Naturwissenschaften Leipzig.

[9] Cowan, R. And Tsang, A. (1994). The falling-leaves mosaic and its equilibrium properties. Adv. Appl. Probab. 26, 54-62. 
[10] Galerne, B. (2011). Computation of the perimeter of measurable sets via their covariogram. Applications to random sets. Image Anal. Stereol. 30, 39-51.

[11] Galerne, B. and Gousseau, Y. (2012). The transparent dead leaves process. Adv. Appl. Probab. 44, 1-20.

[12] Galerne, B. and Lachièze-Rey, R. (2015). Random measurable sets and covariogram realisability problems. Adv. Appl. Probab. 47, 611-639.

[13] Gikhman, I. I. And Skorokhod, A. V. (1974). The Theory of Stochastic Processes I. Classics in mathematics. Springer-Verlag, Berlin.

[14] Hug, D., Last, G. and Weil, W. (2004). A local Steiner-type formula for general closed sets and applications. Math. Z. 246, 237-272.

[15] Ibragimov, I. A. (1995). Remarks on variations of random fields. J. Math. Sci. 75, 1931-1934.

[16] Jeulin, D. (1997). Dead leaves models: from space tesselation to random functions. In Proc. of the Symposium on the Advances in the Theory and Applications of Random Sets. ed. D. Jeulin. World Scientific Publishing Company. pp. $137-156$.

[17] Kingman, J. F. C. (1993). Poisson Processes. Oxford Studies in Probability. The Clarendon Press, Oxford University Press, New York.

[18] LantuéJoul, C. (2002). Geostatistical Simulation: Models and Algorithms. Springer-Verlag, Berlin.

[19] Matheron, G. (1975). Random Sets and Integral Geometry. Wiley series in probability and mathematical statistics. John Wiley \& Sons, New York-LondonSydney.

[20] Meyer, Y. (2001). Oscillating Patterns in Image Processing and Nonlinear Evolution Equations. The Fifteenth Dean Jacqueline B. Lewis Memorial Lectures vol. 22 of Universtity lecture series. American Mathematical Society, Providence, RI. 
[21] Molchanov, I. (2005). Theory of Random Sets. Probability and Its Applications. Springer-Verlag London, Ltd., London.

[22] Rataj, J. (2015). Random sets of finite perimeter. Mathematische Nachrichten 288, 1047-1056.

[23] Rudin, L. I., Osher, S. And Fatemi, E. (1992). Nonlinear total variation based noise removal algorithms. Physica D: Nonlinear Phenomena 60, 259 - 268.

[24] Scheuerer, M. (2010). Regularity of the sample paths of a general second order random field. Stoch. Process. Appl. 120, 1879 - 1897.

[25] Schneider, R. And Weil, W. (2008). Stochastic and Integral Geometry. Probability and Its Applications. Springer-Verlag, Berlin.

[26] Serra, J. (1982). Image Analysis and Mathematical Morphology vol. 1. Academic press, London.

[27] Serra, J., Ed. (1988). Image Analysis and Mathematical Morphology, volume 2 : theoretical advances. Academic press, London.

[28] Stoyan, D. (1986). On generalized planar random tessellations. Math. Nachr. 128, 215-219.

[29] Stoyan, D., Kendall, W. S. and Mecke, J. (1995). Stochastic Geometry and its Applications second ed. Wiley series in probability and mathematical statistics. John Wiley \& Sons, Ltd., Chichester.

[30] Villa, E. (2009). On the outer Minkowski content of sets. Ann. Mat. Pura Appl. 188, 619-630.

[31] Villa, E. (2010). Mean densities and spherical contact distribution function of inhomogeneous Boolean models. Stoch. Anal. Appl. 28, 480-504. 